\title{
New Mixed Ligands with Nickel (II) Complexes, Study of Their Physical \& Spectral Properties, Antibacterial Activities and Laser Irradiation
}

\author{
Zuhoor Fathi Dawood $^{1 *}$; Alaa Jassim Mhommed ${ }^{2}$ \\ ${ }^{1,2}$ Chemistry department, Education College of Pure Science, University of Mosul, Mosul, Iraq \\ Email: ${ }^{1 *}$ pdzfathee@gmail.com, ${ }^{2}$ Alaa.j.m@gmail.com
}

(Received March 20, 2019; Accepted June 11, 2019; Available online March 01, 2020)

DOI: 10.33899/edusj.2020.164361, (C) 2020, College of Education for Pure Science, University of Mosul. This is an open access article under the CC BY 4.0 license (http://creativecommons.org/licenses/by/4.0/).

\begin{abstract}
:
Preparation of two ligands acetonesemicarbazone $(\mathrm{AScH})$ and 2,4-dioximepentane $\left(\mathrm{DO}_{\mathrm{X}} \mathrm{PH}_{2}\right)$ using three ways includes reflux method, heating using microwave rays technique and ultrasonic technique \}, and characterized using physical and spectral techniques. Followed by the preparation of new $\mathrm{Ni}$ (II) complexes containing mixed of three ligands \{including $(\mathrm{AScH}),\left(\mathrm{DO}_{\mathrm{XPH}}\right)$ and 2-ethoxyethanol $\left(\mathrm{EtO}_{2} \mathrm{H}\right)$. The complexes have been characterized using quantitative, physical and spectral techniques. The electrical conductivity indicate that the complexes prepared in slightly neutral medium $(\mathrm{pH}=6-7)$ are electrolytes (1:2), meanwhile the complexes prepared in alkaline medium are nonelectrolytes. All the ligands above acts as bidentate chelating ligands., and octahedral geometries of the complexes are resulted.

The effect of laser have been studied on solid ligands and complexes, no effects have been observed on all the compounds through the results of melting or decomposition point, conductivity and electronic spectra, this means that all the compounds are stable and are not affected by this kind of radiation. Also the biological activity of all the prepared compounds have been evaluated against pathogenic bacterial strains (Staphylococcus aureus, Streptococcus species and E. coli), all the compounds were found to have no antibacterial activity .
\end{abstract}

Keywords: acetonesemicarbazone ,2,4-dioximepentane,2-ethoxy-ethanol, ultrasonic technique, heating using microwave rays, effect of laser, biological activity.

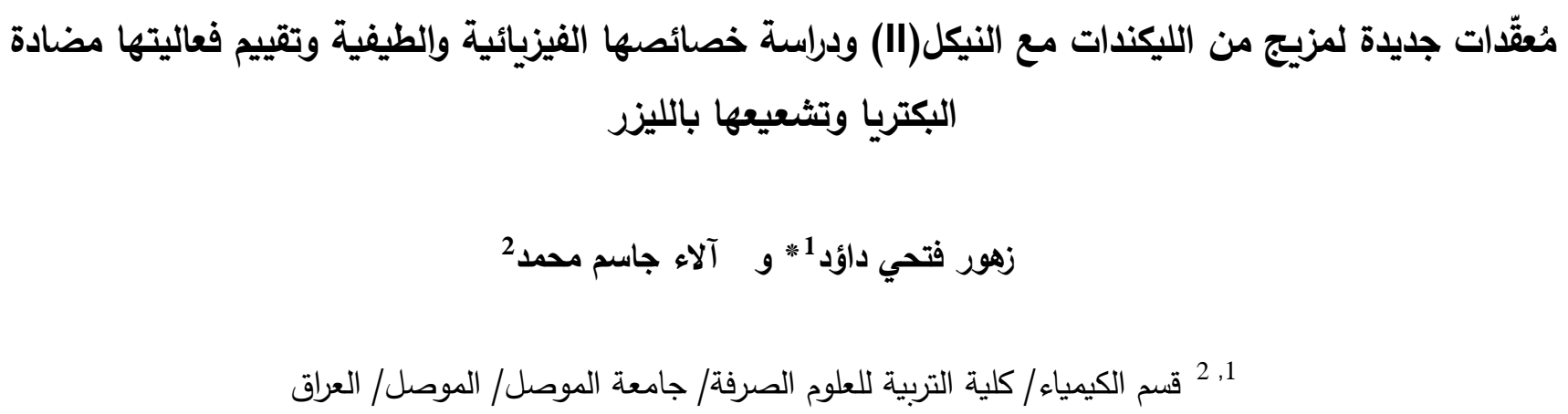




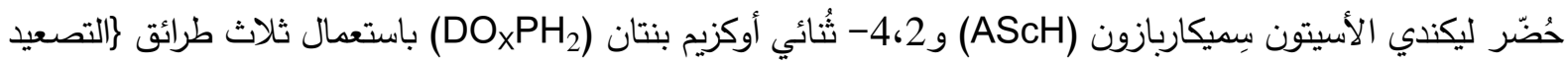

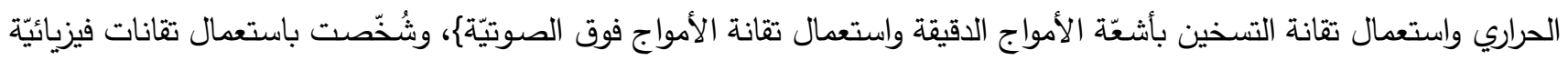
وطيفيّة. أُعقبت بتحضـير مُعقّدات جديدة للنيكل(II) حاويةً على مزيج من ثلاث لتشــل (AScH) و (DOXPH

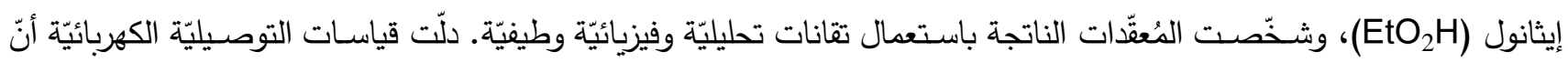

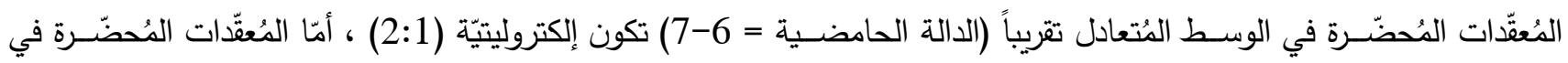
الوسـط القوي فتكون غير إلكتروليتيّة (غير مُوصّـلـة للكهربائيّة). سـلكت جميع الليكندات أعلاه بثـكل ليكندات ثُنائيّة المنح كيليتيّة، ونتجت مُعقّدات ذات أشكال ثُمانيّة السطوح.

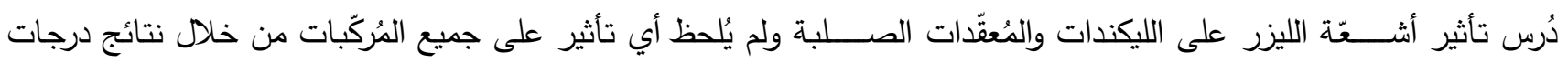

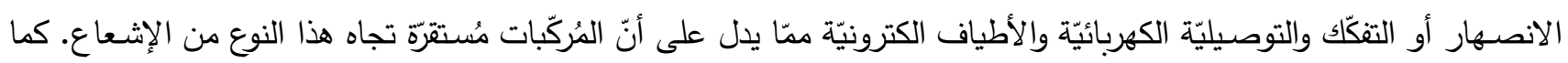

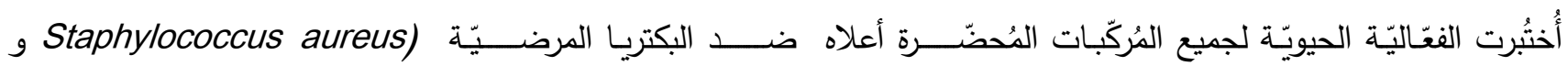
(E. coli و فؤجد أنّ جميعها ليس لها أي تأثير على البكتريا قيد الدرس.

الكلمات الدالة: الأسـيتون سِــيكاربازون ,4 24- ثُنائي أوكزيم بنتان , 2- إيثوكسـي إيثانول , تقانة الأمواج فوق الصــتيّة , تقانة التسخين بأشعَّة الأمواج الدقيقة , تأثير أشعّة الليزر , الفعّاليّة الحيويّة.

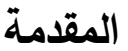

تعد الاختلافات الكبيرة في اساليب التتاسق والأشكال لمُعقّدات النيكل السبب في جعل النيكل يُرّرس بكثافة، فُرست التراكيب

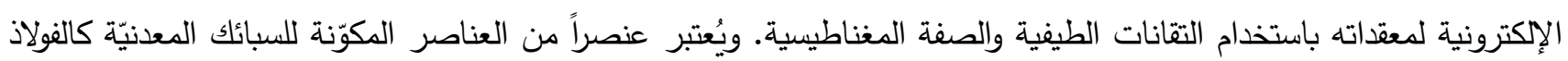
المقاوم للصدأ، وأُستخدمت سبائكه في صناعة المغانيط الدائمة ذات القوّة العالية، وفي صناعة الماكينات الثقيلة والأدوات المستخدمة

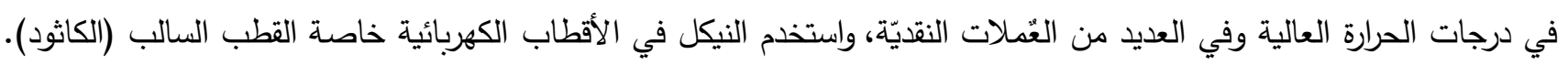
وفي المُجوهرات مع الذهب للحصول على قوّة أفضل وألوان أصليّة، واستخدم في الأعمال الهيكليّة والتغطية الكهربائيّة لمقاومة الصدأ، ولئي

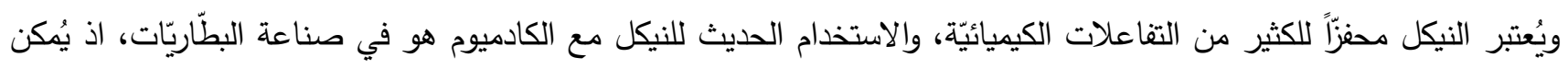

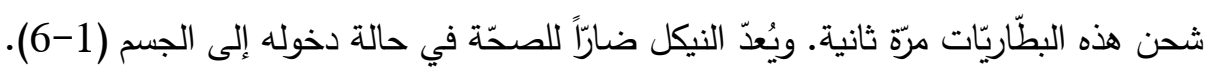

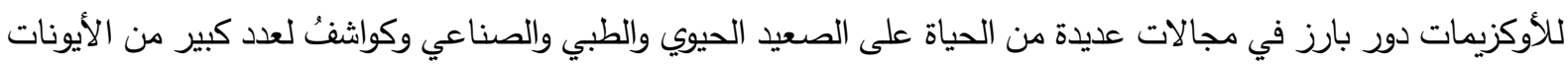

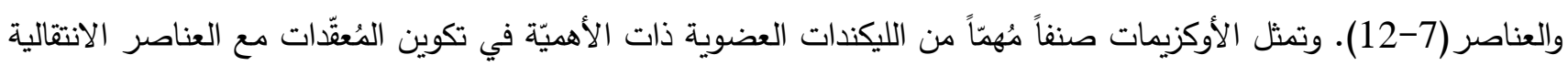
وغير الانتقالية(13-18).

تُمتّل السميكاربازونات صنفاً مُهمّاً من الليكندات العضوية ذات الأهميّة في تكوين المُعقّدات عن طريق منحها للمزدوجات الإكترونية إلى العناصر الفلزية على نحو عام والعناصر الانتقاليّة على وجه خاص، إذ تتناسق من خلال ذرّتي نتروجين الآزوميثين

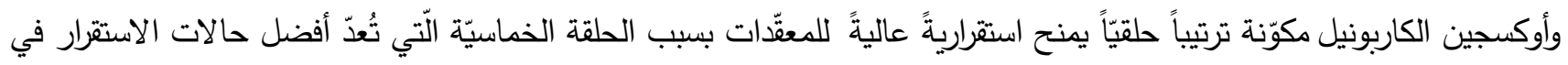
التتاسق فضلاً عن فعّاليتها الحيوية(19-25). يُعد 2-ايثوكسي ايثانول مُذيباً مُستخدماً على نطاق واسع في التطبيقات التجاريّة والصناعيّة. ويسبب الاستنشاق الحاد

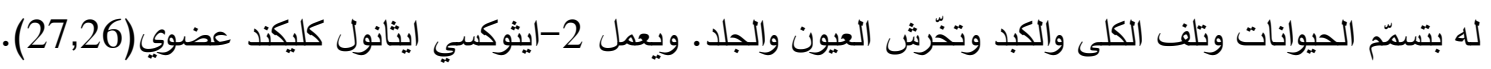

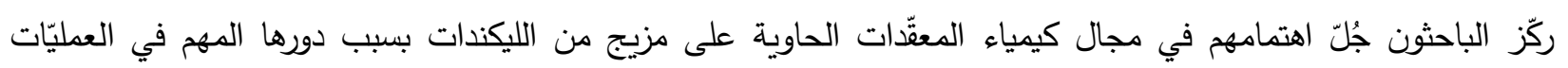
الفيزيولوجيّة والحيويّة، واستتاداً إلى ذلك فإنّ جزءواً كبيراً من الكيمياء التناسقيّة المدوّنة تحتوي على مزيج الليكندات الحاوية على ذرّات 
الكبريت والأوكسجين والنيتروجين مع العناصر الفلزيّة وبحالات تأكسديّة مختلفة(28-30). ونظر اً للأهمية البالغة للمعقّات الحاوية على مزيج من الليكندات فقد حضرت داؤد وجماعتها معقدّدات حاوية على مزيج من الليكندات، وتمّ تشخيصها باستخدام تقانات

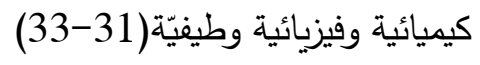

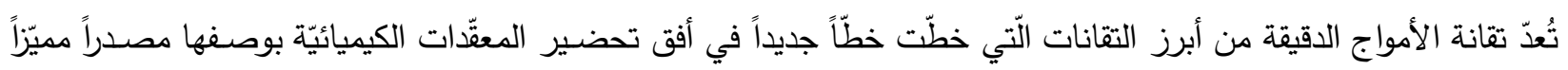
للحرارة. ويُعدّ إثــعاع الأمواج الدقيقة إثــعاعاً كهرومغناطيسـياً يتراوح تردّده مابين

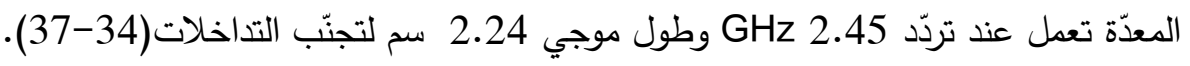

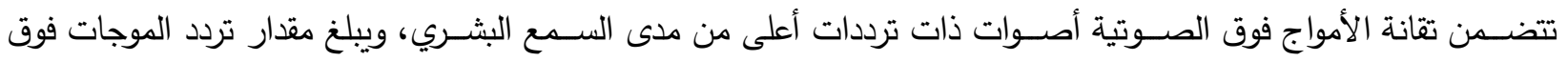

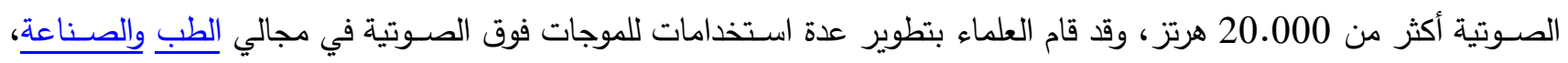

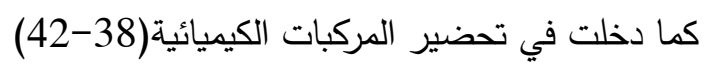
كان لإثعاع الليزر أثر بالغ في الاستخدامات الطبيّة والصناعات الإكترونيّة الدقيقة وغيرها، وتُعدّ تقانة الليزر وسيلة لإنتاج

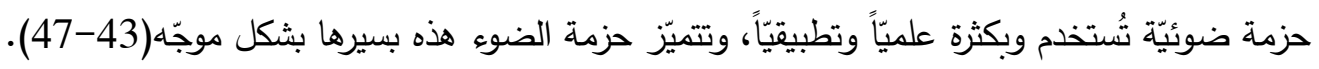

\section{الهذف من البحث}

1- تحضير ليكندات الأوكزيم والسميكاربازون بطرائق التصعيد الحراري أو التسخين بأشعة الأمواج الدقيقة أو الأمواج فوق الصوتية.

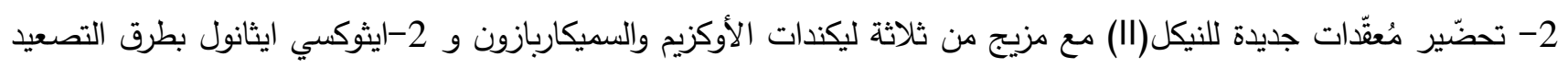

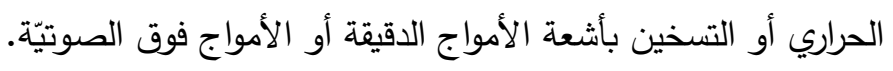

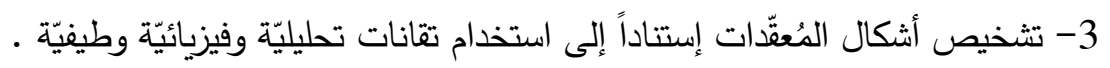
4- مُلاحظة تأثّر الليكندات والمُعقِّات بتسليط أشعّة ليزريّة عليها.

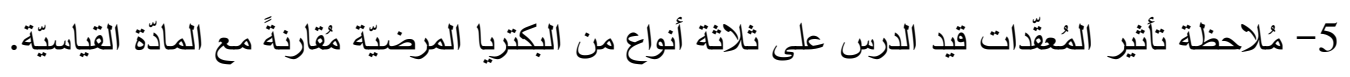

\section{الجزء العملي}

1- المواد الكيميائيّة المُستخدمة

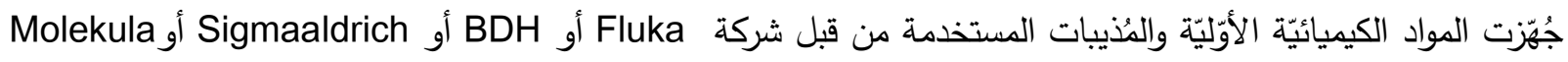

أو ThomasBaker.

2- تحضير الليكندات

حُضّر الليكندان أسيتون سميكاربازون (AScH)(48) (شكل 1) ثم أُعقب بتحضير الليكند 4، 4- ثُنُائي أوكزيم بنتان (48)(DOXPH2) (شكل 1) باستخدام طريقة التصعيد الحراري وبطريقة التسخين بفرن الأمواج الدقيقة كذلك بالأمواج فوق الصوتية.

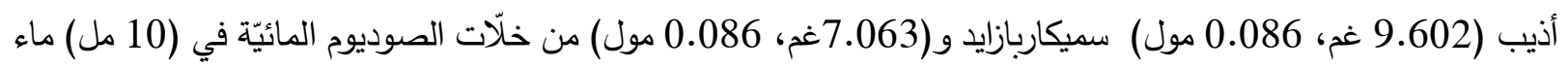
هُقطّر ، وأضيف الأسيتون (5 مل). وحرّك المزيج للحصول على محلول رائق (إذا كان المحلول مُعكّراً سخّن للحصول على محلول

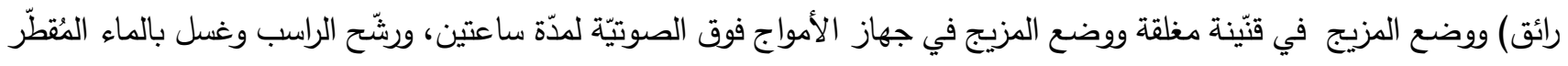

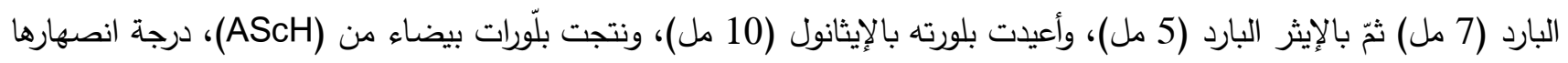
260ْم ووزنها الجُزئي (115.137). 


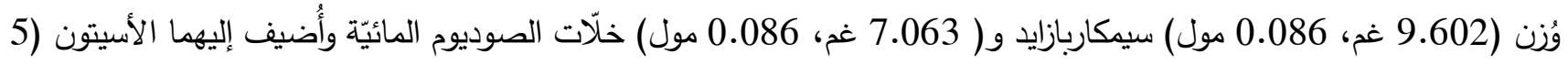

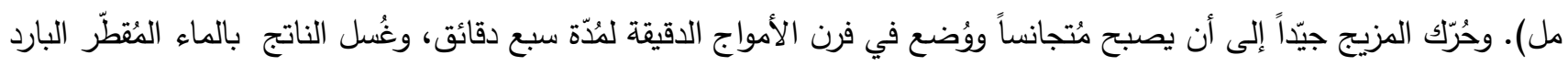

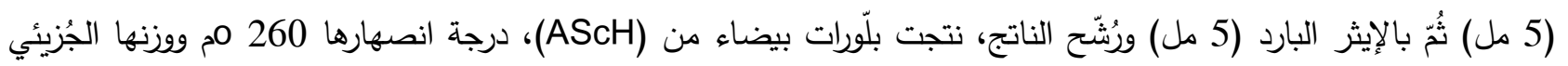

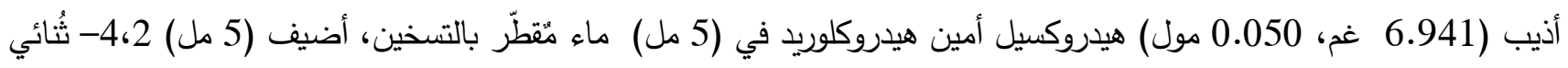

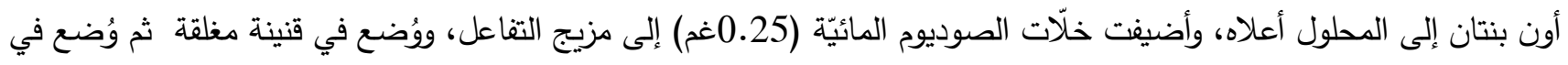

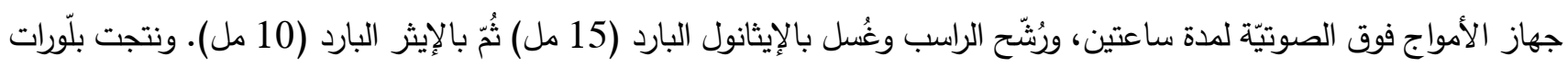

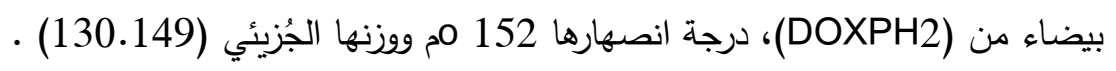

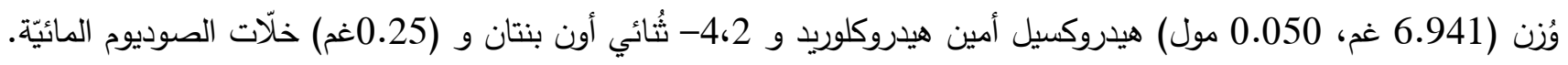

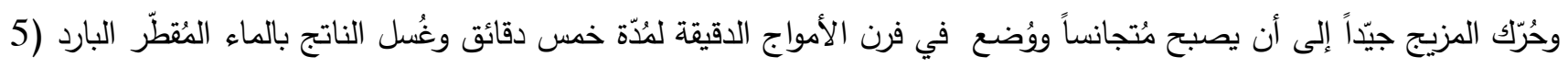

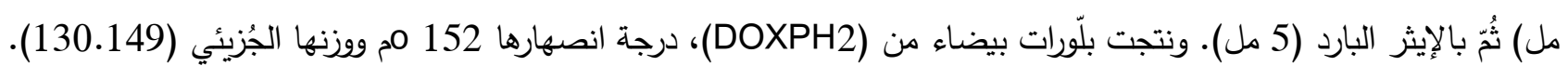
$\mathrm{Me}$
$\prod_{0}$

(AScH) معادلة تحضير الليكند

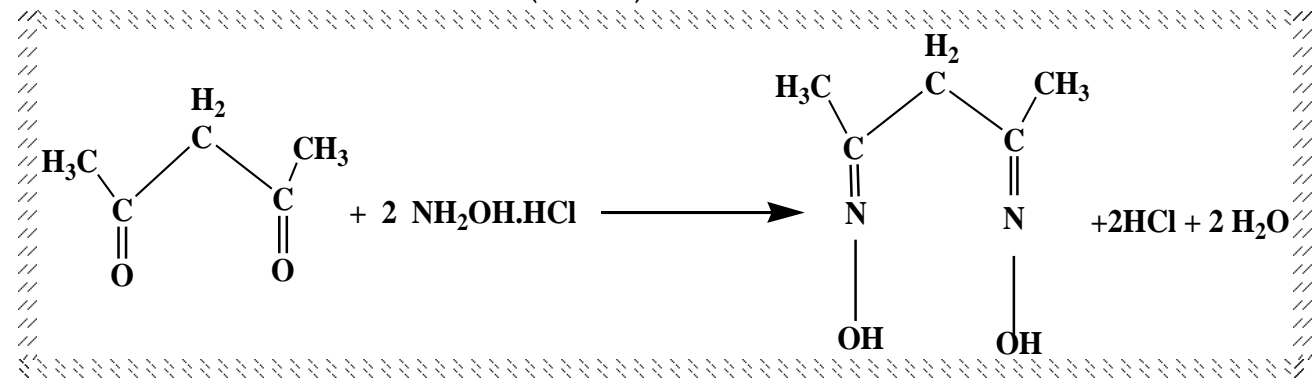

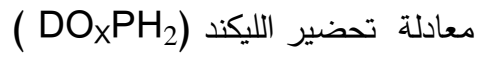

شكل (1) معادلات تحضير الليكندات

3- تحضير المُعقِّات

حُضّرت العديد من المُعقدات عن طريق تفاعل املاح النيكل(II) مع الليكندات في الوسط المتعادل تُقريباً، وأُجري التفاعل

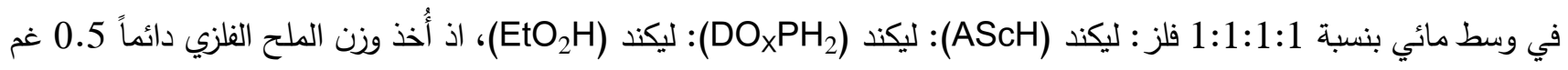

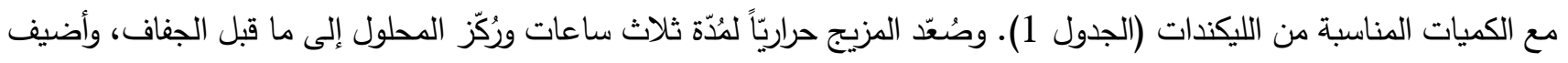

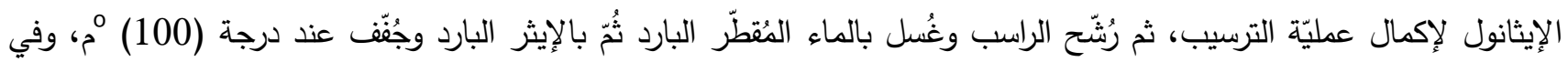

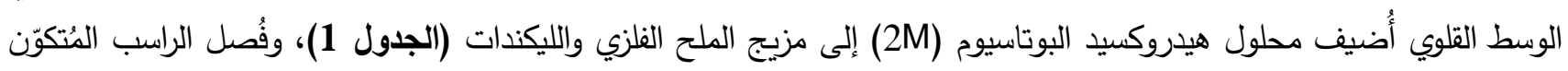

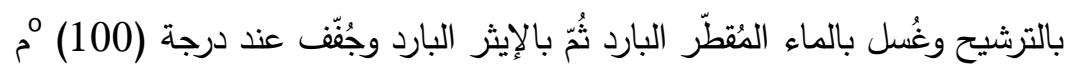

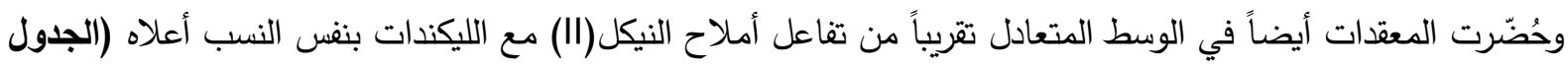

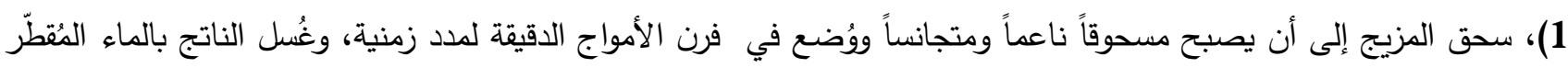

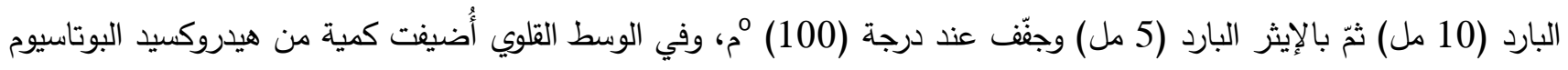




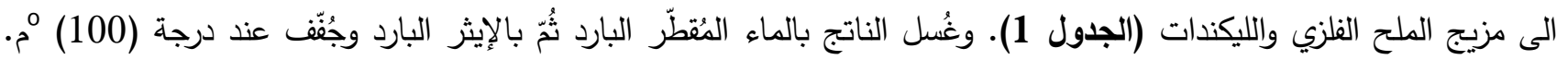
وحُّرت المعقدات أيضاً في الوسط المتعادل تقريباً من تفاعل محاليل أملاح النيكل(II) مع الليكندات، وأُجري التفاعل في وسط مائي بنسبة 1:1:1:1 باستعمال الأمواج فوق الصوتية لمدد زمنية (الجدول 1)، رُكّز المحلول إلى ما قبل الجفاف وأُضيف الإيثانول لإكمال

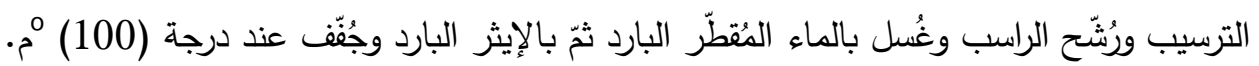

الجدول (1) : أوزان المُركبّات وصيغتها وظروف التفاعل المُستعملة ونسبة الناتج

\begin{tabular}{|c|c|c|c|c|c|c|c|c|c|}
\hline الصيغة المقترحة للمعقدات & $\begin{array}{r}\text { \% } \\
\text { الناتج }\end{array}$ & 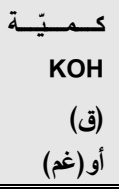 & ون & pH & $\begin{array}{c}\text { وزن } \\
\mathrm{DO}_{x} \mathrm{PH}_{2} \\
\text { (غم) }\end{array}$ & $\begin{array}{c}\text { وزن } \\
\text { (غم) }\end{array}$ & $\begin{array}{c}\text { وزن } \\
\text { EtO }{ }_{2} \mathrm{H} \\
\text { (غم) }\end{array}$ & صيغة الملح & $ت$ \\
\hline$\left[\mathrm{Ni}(\mathrm{ASCH})\left(\mathrm{DO}_{\mathrm{X}} \mathrm{PH}_{2}\right)\left(\mathrm{EtO}_{2} \mathrm{H}\right)\right](\mathrm{Ac})_{2}$ & 96.20 & & & $6-7$ & 0.2615 & 0.2313 & 0.181 & $\mathrm{Ni}\left(\mathrm{CH}_{3} \mathrm{COO}\right)_{2 .} 6 \mathrm{H}_{2} \mathrm{O}$ & 1 \\
\hline$\left[\mathrm{Ni}(\mathrm{ASCH})\left(\mathrm{DO}_{\mathrm{X}} \mathrm{PH}_{2}\right)\left(\mathrm{EtO}_{2} \mathrm{H}\right)\right](\mathrm{Ac})_{2}$ & 76.70 & & 180 & $6-7$ & 0.2615 & 0.2313 & 0.181 & $\mathrm{Ni}\left(\mathrm{CH}_{3} \mathrm{COO}\right)_{2 .} 6 \mathrm{H}_{2} \mathrm{O}$ & 2 \\
\hline$\left[\mathrm{Ni}(\mathrm{ASCH})\left(\mathrm{DO}_{x} \mathrm{PH}_{2}\right)\left(\mathrm{EtO}_{2} \mathrm{H}\right)\right](\mathrm{Ac})_{2}$ & 89.32 & & د4 & $6-7$ & 0.2615 & 0.2313 & 0.181 & $\mathrm{Ni}\left(\mathrm{CH}_{3} \mathrm{COO}\right)_{2 .} 6 \mathrm{H}_{2} \mathrm{O}$ & 3 \\
\hline$\left.\left[\mathrm{Ni}(\mathrm{ASCH})\left(\mathrm{DO}_{x} \mathrm{PH}_{2}\right)\right)\left(\mathrm{EtO}_{2} \mathrm{H}\right)\right]$ & 77.43 & 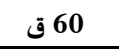 & & 10-11 & 0.2615 & 0.2313 & 0.181 & $\mathrm{Ni}\left(\mathrm{CH}_{3} \mathrm{COO}\right)_{2 .} 6 \mathrm{H}_{2} \mathrm{O}$ & 4 \\
\hline$\left.\left[\mathrm{Ni}(\mathrm{ASCH})\left(\mathrm{DO}_{x} \mathrm{PH}_{2}\right)\right)\left(\mathrm{EtO}_{2} \mathrm{H}\right)\right]$ & 91.14 & $\begin{array}{c}0.337 \\
\text { غم }\end{array}$ & 6 & $10-11$ & 0.2615 & 0.2313 & 0.181 & $\mathrm{Ni}\left(\mathrm{CH}_{3} \mathrm{COO}\right)_{2 .} 6 \mathrm{H}_{2} \mathrm{O}$ & 5 \\
\hline$\left[\mathrm{Ni}(\mathrm{ASCH})\left(\mathrm{DO}_{x} \mathrm{PH}_{2}\right)\left(\mathrm{EtO}_{2} \mathrm{H}\right)\right] \mathrm{Cl}_{2}$ & 94.98 & & & $6-7$ & 0.273 & 0.242 & 0.190 & $\mathrm{Ni} \mathrm{Cl} 2.6 \mathrm{H}_{2} \mathrm{O}$ & 6 \\
\hline$\left[\mathrm{Ni}(\mathrm{ASCH})\left(\mathrm{DO}_{x} \mathrm{PH}_{2}\right)\left(\mathrm{EtO}_{2} \mathrm{H}\right)\right] \mathrm{Cl}_{2}$ & 69.39 & & 180 & $6-7$ & 0.273 & 0.242 & 0.190 & $\mathrm{Ni} \mathrm{Cl} 2.6 \mathrm{H}_{2} \mathrm{O}$ & 7 \\
\hline$\left[\mathrm{Ni}(\mathrm{ASCH})\left(\mathrm{DO}_{\mathrm{x}} \mathrm{PH}_{2}\right)\left(\mathrm{EtO}_{2} \mathrm{H}\right)\right] \mathrm{Cl}_{2}$ & 97.96 & & $د 2$ & 6-7 & 0.273 & 0.242 & 0.190 & $\mathrm{Ni} \mathrm{Cl} 2.6 \mathrm{H}_{2} \mathrm{O}$ & 8 \\
\hline$\left[\mathrm{Ni}(\mathrm{ASCH})\left(\mathrm{DO}_{x} \mathrm{PH}_{2}\right)\left(\mathrm{EtO}_{2} \mathrm{H}\right)\right]$ & 76.38 & ما & & 10-11 & 0.273 & 0.242 & 0.190 & $\mathrm{Ni} \mathrm{Cl} 2.6 \mathrm{H}_{2} \mathrm{O}$ & 9 \\
\hline$\left[\mathrm{Ni}(\mathrm{ASCH})\left(\mathrm{DO}_{\mathrm{x}} \mathrm{PH}_{2}\right)\left(\mathrm{EtO}_{2} \mathrm{H}\right)\right]$ & 97.56 & $\begin{array}{r}0.505 \\
\text { غم }\end{array}$ & $د \mathbf{3}$ & $10-11$ & 0.273 & 0.242 & 0.190 & $\mathrm{Ni} \mathrm{Cl} 2.6 \mathrm{H}_{2} \mathrm{O}$ & 10 \\
\hline$\left[\mathrm{Ni}(\mathrm{ASCH})\left(\mathrm{DO}_{x} \mathrm{PH}_{2}\right)\left(\mathrm{EtO}_{2} \mathrm{H}\right)\right]\left(\mathrm{SO}_{4}\right)_{2}$ & 84.79 & & & $6-7$ & 0.232 & 0.198 & 0.160 & $\mathrm{Ni} \mathrm{SO}{ }_{4} \cdot 6 \mathrm{H}_{2} \mathrm{O}$ & 11 \\
\hline$\left[\mathrm{Ni}(\mathrm{ASCH})\left(\mathrm{DO}_{x} \mathrm{PH}_{2}\right)\left(\mathrm{EtO}_{2} \mathrm{H}\right)\right]\left(\mathrm{SO}_{4}\right)_{2}$ & 51.72 & & 180 & $6-7$ & 0.232 & 0.198 & 0.160 & $\mathrm{Ni} \mathrm{SO}{ }_{4} .6 \mathrm{H}_{2} \mathrm{O}$ & 12 \\
\hline$\left[\mathrm{Ni}(\mathrm{ASCH})\left(\mathrm{DO}_{X} \mathrm{PH}_{2}\right)\left(\mathrm{EtO}_{2} \mathrm{H}\right)\right]\left(\mathrm{SO}_{4}\right)_{2}$ & 97.70 & & $د 3$ & 6-7 & 0.232 & 0.198 & 0.160 & $\mathrm{Ni} \mathrm{SO}{ }_{4} .6 \mathrm{H}_{2} \mathrm{O}$ & $\begin{array}{l}1 \\
3\end{array}$ \\
\hline$\left[\mathrm{Ni}(\mathrm{ASCH})\left(\mathrm{DO}_{x} \mathrm{PH}_{2}\right)\left(\mathrm{EtO}_{2} \mathrm{H}\right)\right]$ & 70.19 & 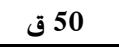 & & $8-9$ & 0.232 & 0.198 & 0.160 & $\mathrm{Ni} \mathrm{SO}{ }_{4} .6 \mathrm{H}_{2} \mathrm{O}$ & 14 \\
\hline$\left[\mathrm{Ni}(\mathrm{ASCH})\left(\mathrm{DO}_{\mathrm{X}} \mathrm{PH}_{2}\right)\left(\mathrm{EtO}_{2} \mathrm{H}\right)\right]$ & 98.57 & $\begin{array}{c}0.282 \\
\dot{~}\end{array}$ & $د 4$ & $8-9$ & 0.232 & 0.198 & 0.160 & $\mathrm{Ni} \mathrm{SO}{ }_{4} .6 \mathrm{H}_{2} \mathrm{O}$ & 15 \\
\hline
\end{tabular}

*ن تعني زمن التقاعل بالأمواج فوق الصوتية ون * تعني زمن التشعيع بفرن الأمواج الدقيقة وق قطرة وpH الدالة حامضية .

4- القياسات التحليلية والفيزيائية والطيفية والفعالية الحيوية :

تمّ حساب كميّة الكاربون والهيدروجين والنتروجين والكبريت (CHNS) بجهاز في مركز البحوث العلميّة والتطبيقيّة نوع

- EuroE3000/Italy إيطالي المنشأ من نوع : Instruments Elemental Combustion Costech موديل 4010. كما تّ حساب كميّة النيكل(49) بجهاز مطياف الامتصاص الذربي من نوع Sens AAGBC Scientific Equipment في المعهد التقني الطبّي - جامعة التقنيّة الثماليّة. وعُيتنت كميّة الكلور باستعمال الطريقة الحجميّة (التسحيح)(49)، وقيست درجات الإنصهار أو التفكّك لليكندات ومُعقّداتها المُحضّرة

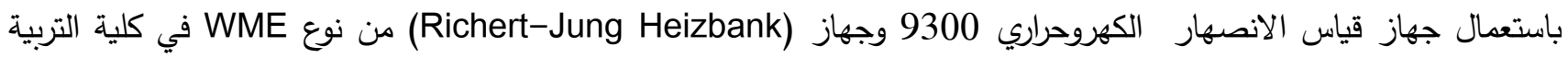

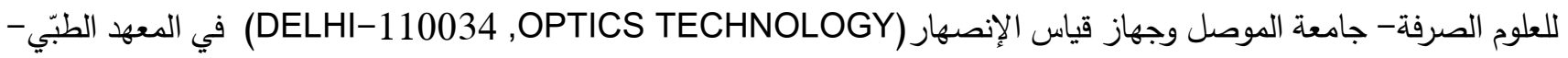
(Multiline F/SET- جامعة التقنيّة الثماليّة. وقيست التوصيليّة الكهبائيّة للمُعقدّات المُحضّرة باستعمال جهازي التوصيل الكهربائي (Conductivity Meter-Model LF- g EWTW Wiscenschaf Technische Werketattem 82362 Weinheim) (42 باستعمال مُذيب ثُائي مثيل فورماميد وبتركيز 3-10 مولاري بدرجة حرارة 25 م. مقيست المغناطيسيّة للمُعقّدات المُحضّرة عند 
درجة حرارة 25 o في كلية التربية للعلوم الصرفة- جامعة الموصل باستعمال طريقة فَرادي بجهاز صمّمه (D.F. Evans)

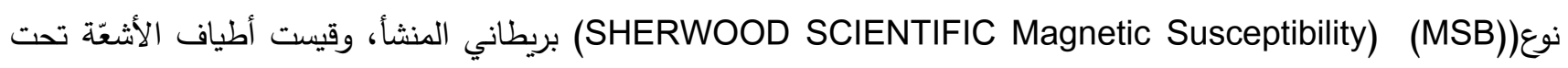
الحمراء لليكندات والمُعقّدات المُحضّرة بجهاز من نوع Model Alpha-Bruker الألماني المنشأ المُتّواجد في كلية التربية - جامعة وفئية

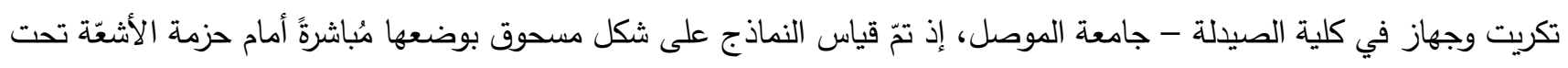

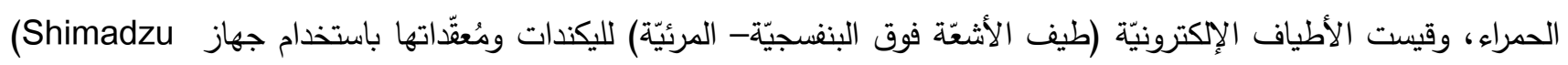
UV-1650 PC UV-Visible Spectrophotometer) 1 مولاري وباستعمال خلايا الكوارتز ذات مسار 1 سم في المدى 200-1100 نانومتر وفي درجة حرارة 25 م. م. وقيس طيف الكتلة باستعمال جهاز Water-Micromass LCT Electrospray Mass Spectrometer جامعة طهران - ايران.

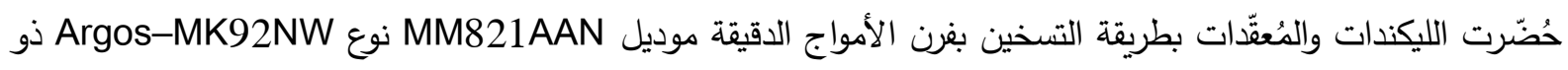

قدرة (500 واط)، وكذلك بجهاز الأمواج فوق الصوتية في كلية التربية للعلوم الصرفة- جامعة الموصل. شُعْعت الليكندات والمُعقدّات

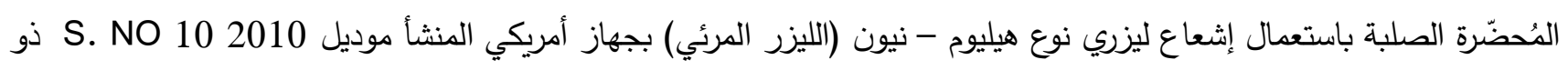
قدرة 1 ملي واط وطول موجي 600-700 نانومتر ولفترات زمنيّة تراوحت بين نصف ساعة إلى ساعتين.

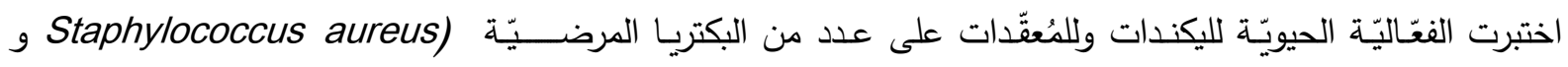
باستخدام تقانة الانتشار على سطح الآكار كمضادّات للبكتريا من قبل قسم علوم الحياة- (E. coli Streptococcus specie) كليّة العلوم- جامعة الموصل.

\section{النتائج وإلمناقشة}

يُككن التعبير عن تكوّن مُعقّات النيكل(II) قيد الدرس والمُحضّــرة بطريقة التصـعيد الحراري وطريقة الأمواج فوق الصــوتيّة

وبطريقة التسخين بأشعّة الأمواج الدقيقة في كلّ من الوسطين المُتعادل تقريباً والقلوي كما في اليُعادلات الآتبة: $\mathrm{NiX}_{\mathrm{n}} \cdot 6 \mathrm{H}_{2} \mathrm{O}+\mathrm{AScH}+\mathrm{DOxPH} \mathrm{H}_{2}+\mathrm{EtO}_{2} \mathrm{H} \rightarrow\left[\mathrm{Ni}(\mathrm{AScH})\left(\mathrm{DOxPH}_{2}\right)\left(\mathrm{EtO}_{2} \mathrm{H}\right)\right] \mathrm{X}_{\mathrm{n}}+6 \mathrm{H}_{2} \mathrm{O}$ $\mathrm{NiSO}_{4} .6 \mathrm{H}_{2} \mathrm{O}+\mathrm{AScH}+\mathrm{DOxPH}+\mathrm{EtO}_{2} \mathrm{H} \rightarrow\left[\mathrm{Ni}(\mathrm{AScH})\left(\mathrm{DOxPH}_{2}\right)\left(\mathrm{EtO}_{2} \mathrm{H}\right)\right]+6 \mathrm{H}_{2} \mathrm{O}$ $\mathrm{NiX} \cdot 6 \mathrm{H}_{2} \mathrm{O}+\mathrm{AScH}+\mathrm{DOxPH}+\mathrm{EtO}_{2} \mathrm{H}+\mathrm{KOH} \rightarrow\left[\mathrm{Ni}(\mathrm{AScH})(\mathrm{DOxPH})\left(\mathrm{EtO}_{2}\right)\right]+2 \mathrm{KX}_{\mathrm{n}}+8 \mathrm{H}_{2} \mathrm{O}$ $\mathrm{NiSO}_{4} \cdot 6 \mathrm{H}_{2} \mathrm{O}+\mathrm{AScH}+\mathrm{DOxPH}+\mathrm{EtO}_{2} \mathrm{H}+\mathrm{KOH} \rightarrow\left[\mathrm{Ni}(\mathrm{AScH})(\mathrm{DOxPH})\left(\mathrm{EtO}_{2}\right)\right]+\mathrm{K}_{2} \mathrm{SO}_{4}+8 \mathrm{H}_{2} \mathrm{O}$ (2= حيث أثبتت النتائج التحليليّة بأنّ كميّة النيكل والكلور كانت مُتَّقة مع الصـــيخ المُقترحة كما أنّ كميّة الكاربون والهيدروجين والنتروجين والكبريت والمُدوّنة في الجدول رقم (2)، كانت مُتِققة أيضاً مع الصيخ المُقترحة. 
الجدول (2): النتائج الكميّة للعناصر في المُعقدّات (عمبات

\begin{tabular}{|c|c|c|c|c|c|c|}
\hline $\begin{array}{c}\text { \%Ni } \\
\text { (عمليا)نريا }\end{array}$ & \%Cl (عمليا)نظريا & \% \% (عليا)نظريا & \% (عمليانظريا & \%H (عمليا)نظريا & (عمليا)نظريا & $ت$ \\
\hline$(11.43) 11.46$ & - & - & 13.67 & 6.88 & 39.87 & 1 \\
\hline$(11.42) 11.46$ & - & - & $(13.63) 13.67$ & $(6.81) 6.88$ & (39.79)39.87 & 2 \\
\hline (11.41)11.46 & - & - & 13.67 & 6.88 & $\mathbf{3 9 . 8 7}$ & 3 \\
\hline (14.94)14.97 & - & - & $(17.82) 17.86$ & $(6.90) 6.94$ & (39.77) 39.82 & 4 \\
\hline$(14.92) 14.97$ & - & - & 17.86 & 6.94 & 39.82 & 5 \\
\hline$(12.57) 12.62$ & $(15.20) 15.26$ & - & $(14.99) 15.05$ & $(6.21) 6.28$ & $(33.52) 33.55$ & 6 \\
\hline$(12.58) 12.62$ & $(15.15) 15.26$ & - & $(14.99) 15.05$ & $(6.24) 6.28$ & $(33.50) 33.55$ & 7 \\
\hline$(12.58) 12.62$ & $(15.10) 15.26$ & - & 15.05 & 6.28 & $\mathbf{3 3 . 5 5}$ & 8 \\
\hline$(14.93) 14.97$ & - & - & 17.86 & 6.94 & 39.82 & 9 \\
\hline (14.92)14.97 & - & - & $(\mathbf{1 7 . 8 3}) 17.86$ & $(6.87) 6.94$ & $(39.75) 39.82$ & 10 \\
\hline (11.91)11.98 & - & $(6.49) 6.54$ & $(14.22) 14.29$ & (5.91)5.96 & (31.81) 31.86 & 11 \\
\hline (11.94)11.98 & - & 6.54 & $(14.20) 14.29$ & 5.96 & 31.86 & 12 \\
\hline$(11.93) 11.98$ & - & $(6.47) 6.54$ & $(14.23) 14.29$ & (5.89) 5.96 & $(31.78) 31.86$ & 13 \\
\hline$(14.93) 14.97$ & - & - & 17.86 & 6.94 & 39.82 & 14 \\
\hline (14.91)14.97 & - & - & $(17.82) 17.86$ & $(6.88) 6.94$ & (39.76) 39.82 & 15 \\
\hline
\end{tabular}

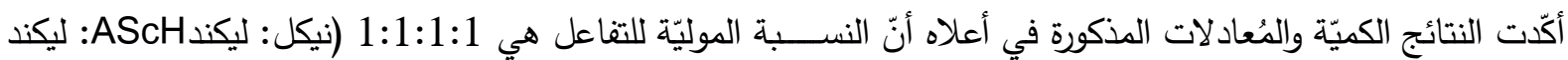

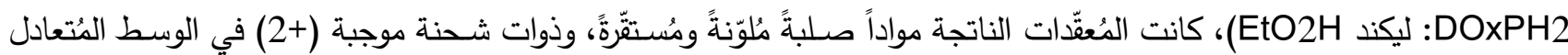

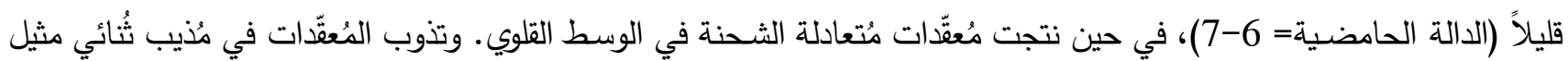

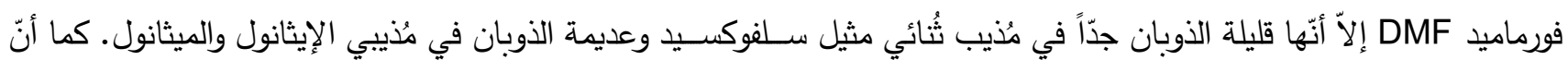

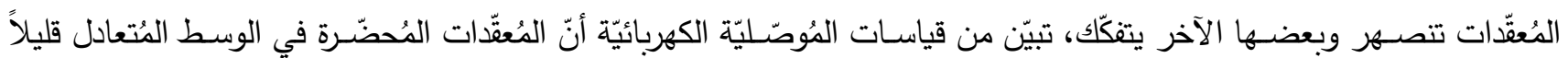
(الدالة الحامضية= 6-7) تكون مُوصّلة إلكتروليتيّة بنسبة 2:1 (50)، في حين تسلك المُعقّدات المُحضّرة في الوسط القلوي سلوك

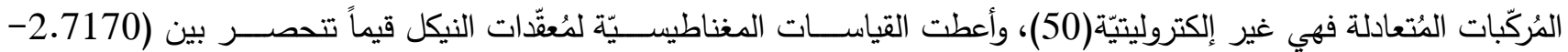
3.2596) بور مغناطون، ممّا يُرجّح كون ذرّة النيكل سُداسيّة التناسق ذات شكل ثُمَاني السطوح عالي البرم(51).

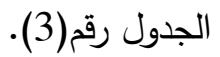


الجدول (3) : الصفات الفيزيائيّة للمُعقّدات المُحضّرة

\begin{tabular}{|c|c|c|c|c|c|}
\hline M.wt** & $\begin{array}{c}\mu_{\text {eff }} \\
(\text { B.M) }\end{array}$ & $\Lambda_{M}{ }^{*}$ & لدرجة الإنصهُار التفكار) & اللون & $ت$ \\
\hline 512.2126 & 3.2444 & 155 & $195 *$ & اخضر فاتح & 1 \\
\hline 512.2126 & 3.2305 & 156 & 195* & اخضر فاتح & 2 \\
\hline 512.2126 & 3.2159 & 154 & 195* & اخضر فاتح & 3 \\
\hline 392.1052 & 3.2112 & 2 & 280 & زيتوني فاتح & 4 \\
\hline 392.1052 & 3.1997 & 3 & 280 & زيتوني فاتح & 5 \\
\hline 465.3726 & 3.2596 & 169 & 200* & اخضر مصفز & 6 \\
\hline 465.3726 & 3.3080 & 170 & $200^{*}$ & اخضر مصفز & 7 \\
\hline 465.3726 & 3.2147 & 168 & 200* & اخضر مصفر & 8 \\
\hline 392.1052 & 3.2112 & 7 & 264 & زيتوني فاتح & 9 \\
\hline 392.1052 & 3.2440 & 6 & 264 & زيتوني فاتح & 10 \\
\hline 490.1729 & 3.2006 & 163 & 243 & اخضر فاتح & 11 \\
\hline 490.1729 & 3.1879 & 162 & 243 & اخضر فاتح & 12 \\
\hline 490.1729 & 3.2161 & 162 & 243 & اخضر فاتح & 13 \\
\hline 392.1052 & 3.2338 & 2 & 264 & زيتوني فاتح & $\overline{14}$ \\
\hline 392.1052 & 3.2444 & 3 & 264 & زيتوني فاتح & 15 \\
\hline
\end{tabular}

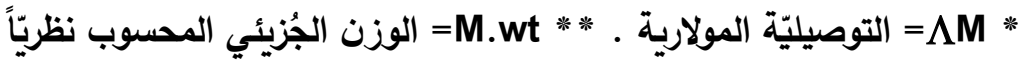

\section{الأطياف فوق البنفسيّة - المرئيّة}

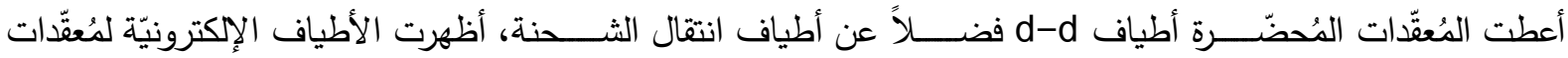

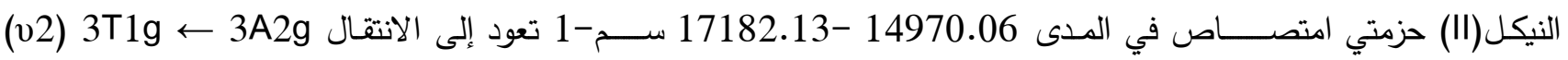

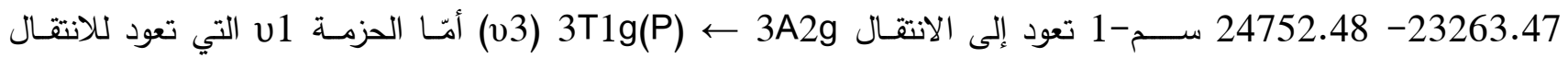

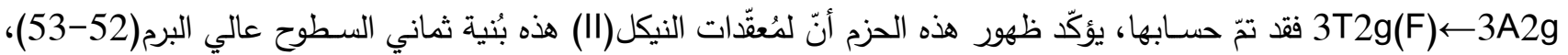

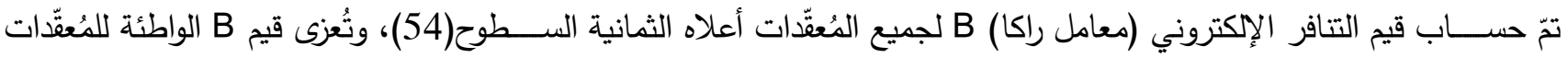

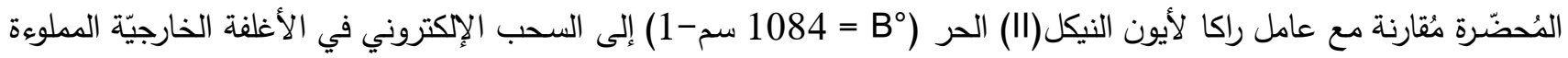

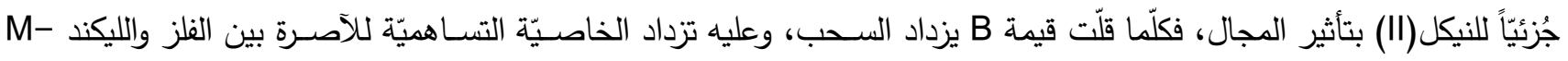

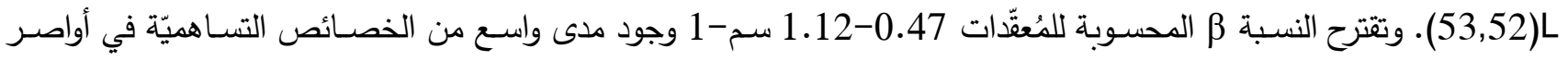

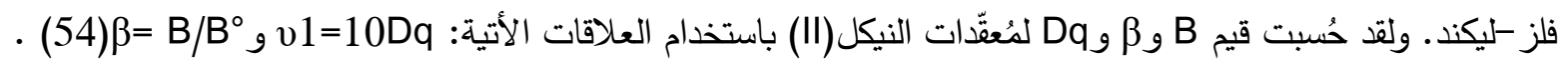


الجدول (4): نتائج الطيف فوق البنفسجي - المرئي وعوامل راكا

\begin{tabular}{|c|c|c|c|c|c|c|c|c|c|c|c|c|}
\hline $\begin{array}{r}\text { C.F.S.E } \\
\left(\mathrm{cm}^{-1}\right)\end{array}$ & $\beta$ & $v_{3} / v_{2}$ & $v_{3} / v_{1}$ & $v_{2} / v_{1}$ & $\mathrm{Dq} / \mathbf{B}$ & $\begin{array}{l}\text { 10Dq } \\
\left(\mathrm{cm}^{-1}\right)\end{array}$ & $\begin{array}{c}\text { B } \\
\left(\mathrm{cm}^{-1}\right)\end{array}$ & $\begin{array}{c}\mathbf{C} . \mathbf{T} \\
\left(\mathbf{c m}^{-1}\right)\end{array}$ & $\begin{array}{c}v_{3} \\
\left(\mathbf{c m}^{-1}\right)\end{array}$ & $\begin{array}{c}v_{2} \\
\left(\mathbf{c m}^{-1}\right)\end{array}$ & $\begin{array}{c}v_{1} \\
\left(\mathbf{c m}^{-1}\right)\end{array}$ & المعقد \\
\hline 12232.42 & 0.59 & 1.57 & 2.40 & 1.53 & 1.6 & 1019.37 & 635.39 & 36496.35 & 24430.57 & 15600.62 & 10193.68 & 1 \\
\hline 12232.42 & 0.59 & 1.57 & 2.40 & 1.53 & 1.6 & 1019.37 & 635.39 & 36496.35 & 24430.57 & 15600.62 & 10193.68 & 2 \\
\hline 12232.42 & 0.59 & 1.57 & 2.40 & 1.53 & 1.6 & 1019.37 & 635.39 & 36496.35 & & 15600.62 & 10193.68 & 3 \\
\hline 12207.53 & 0.47 & 1.55 & 2.28 & 1.47 & 2.0 & $101^{\prime}$ & 509.72 & 37037.04 & & 14970 & & 4 \\
\hline 12207.53 & 0.47 & 1.55 & 2.28 & 1.47 & 2.0 & & 509.72 & 37037.04 & 2326 & 1497 & 2.94 & 5 \\
\hline 12232 & 1.12 & 1.44 & 3.16 & 2.16 & 0.8 & & 1213.36 & & & 171 & & 6 \\
\hline 12232.42 & 1.12 & 1.44 & 3.16 & 2.16 & 0.8 & 1019 & 1213.36 & 3636 & 2475 & 171 & 3.68 & 7 \\
\hline 12232.42 & 1.12 & 1.44 & 3.16 & 2.16 & 0.8 & 1014 & 1213.36 & 36363.64 & 2475 & 1718 & 3.68 & 8 \\
\hline 12207.53 & 0.47 & 1.56 & 2.28 & 1.47 & 2.0 & 1017.29 & 509.72 & 37037.04 & 23263 & 14970.06 & 10172.94 & 9 \\
\hline 12207.53 & 0.47 & 1.56 & 2.28 & 1.47 & 2.0 & 1017.29 & 509.72 & 37037.04 & 23263.47 & 14970.06 & 10172.94 & 10 \\
\hline 12121.21 & 0.59 & 1.57 & 2.40 & 1.54 & 1.5 & 1010.10 & 663.41 & 37037.03 & 24354.46 & 15552.10 & 10101.01 & 11 \\
\hline 12121.21 & 0.59 & 1.57 & 2.40 & 1.54 & 1.5 & 1010.10 & 663.41 & 37037.03 & 24354.46 & 15552.10 & 10101.01 & 12 \\
\hline 12121.21 & 0.59 & 1.57 & 2.40 & 1.54 & 1.5 & 1010.10 & 663.41 & 37037.03 & 24354.46 & 15552.10 & 10101.01 & 13 \\
\hline 12207.53 & 0.47 & 1.55 & 2.28 & 1.47 & 2.0 & 1017.29 & 509.72 & 37037.04 & & 14970.06 & 10172.94 & 14 \\
\hline 12207.53 & 0.47 & 1.55 & 2.28 & 1.47 & 2.0 & 1017.29 & 509.72 & 37037.04 & 23263.47 & 14970.06 & 10172.94 & 15 \\
\hline
\end{tabular}
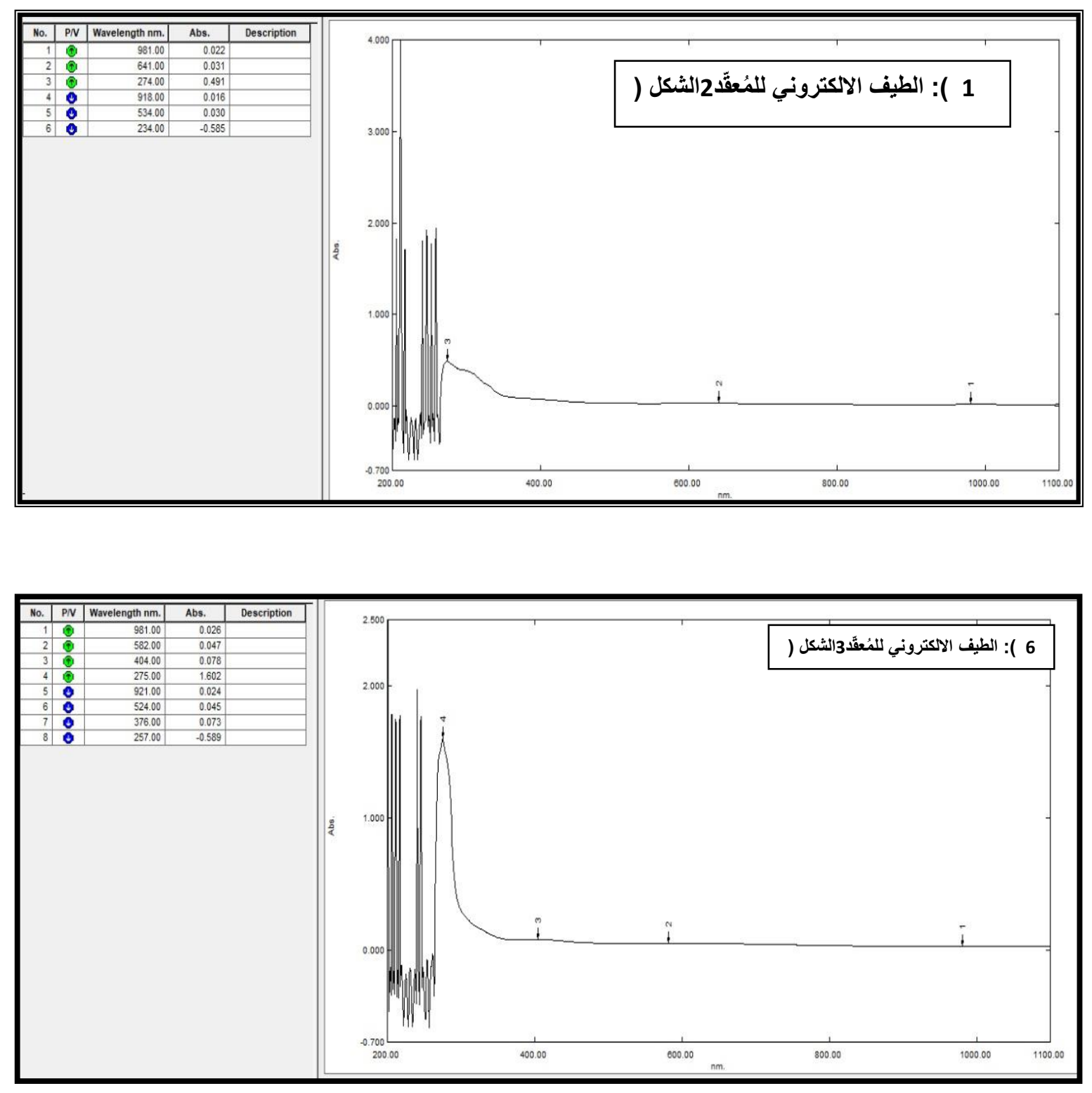
أطياف الأشقّة تحت الحمراء

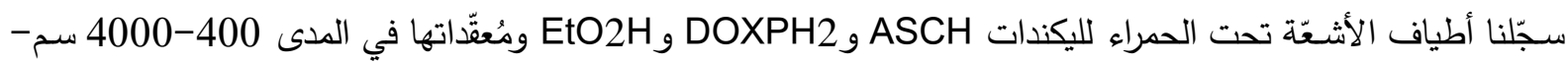
1، والجدول(6) يُبيّن قيم الحزم ذات العلاقة ومواقعها في هذه الأطياف. تمّت دراســة مواقع التتاسـق لليكندات وتكوّن الأواصـر بين

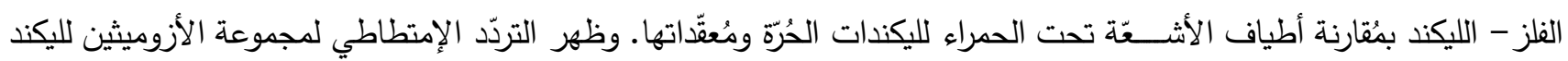

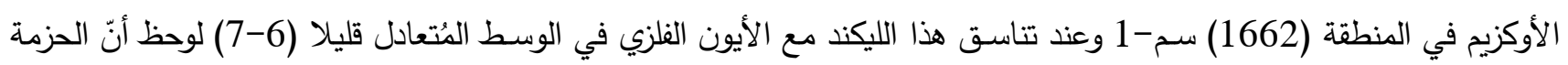

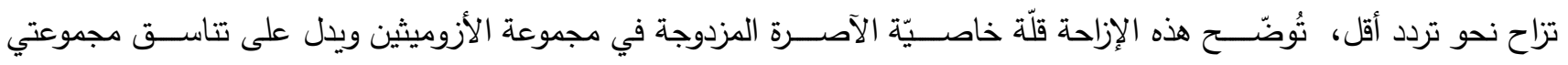

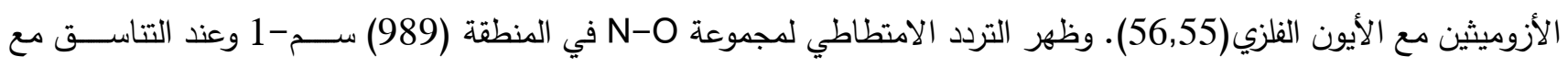

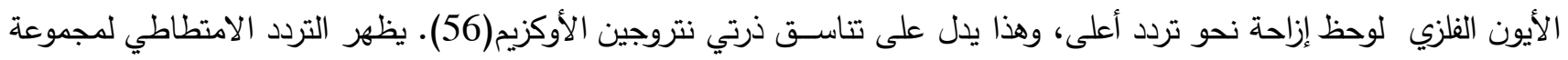

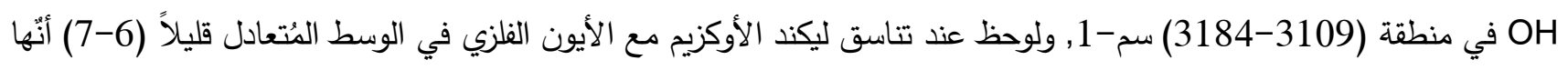

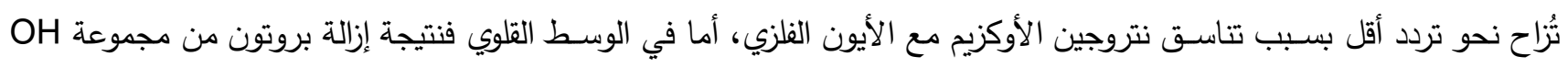

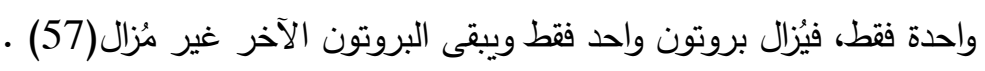

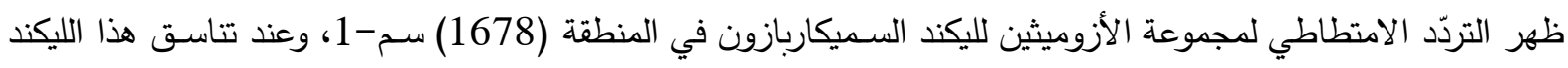

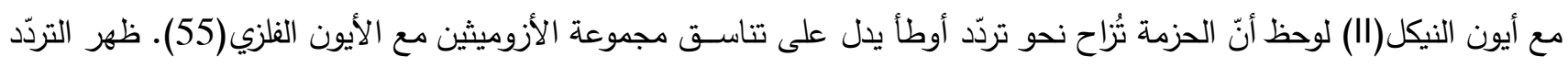

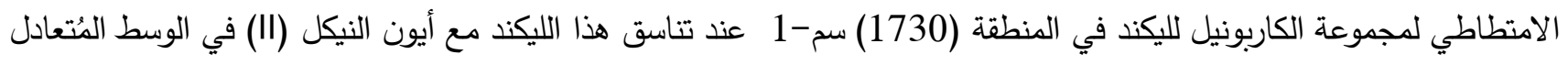

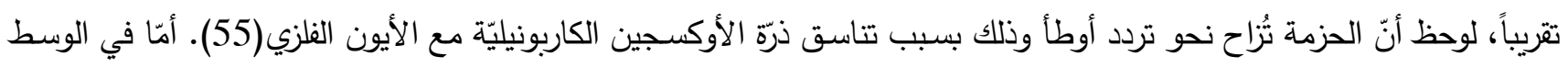

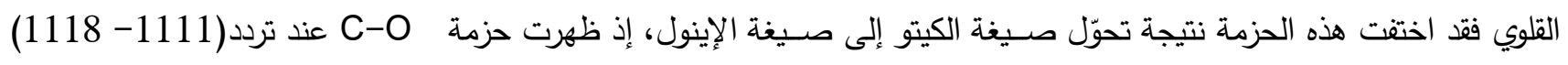

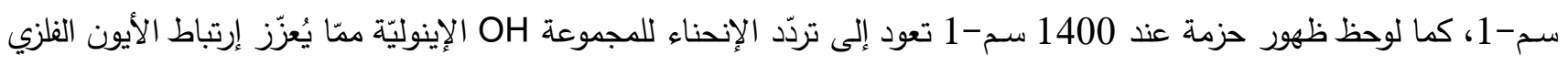

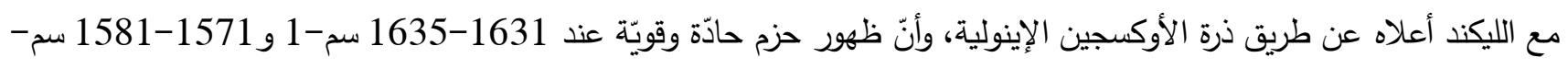

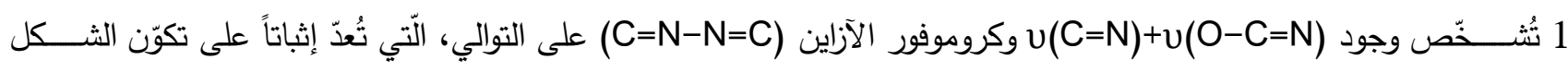

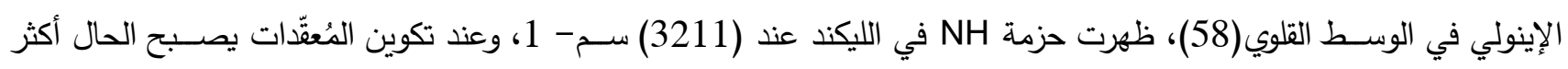
تعقيداً بسبب العديد من العوامل(55) منها: تأثير الآصرة الهيدروجينيّة (يحدث عادة إنكسار بالآصرة الهيدروجينية عند التتاسق مُسبّباً

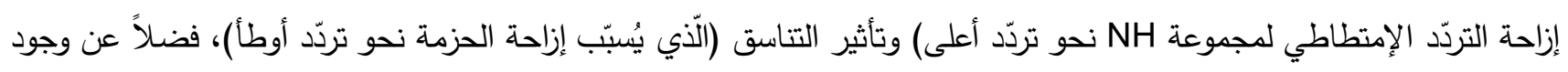

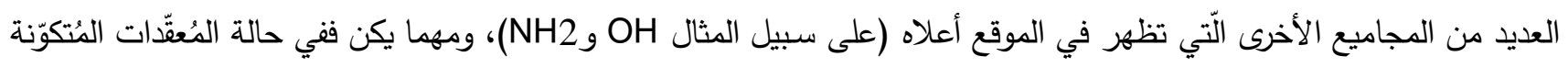
في الوســ المُتعادل تَظهر حزمة NH في الموقع نفسـهـ تقريباً، ومن المتوقع عدم وجود تناسـق بين ذرّة نتروجين الأميدو مع الأيون

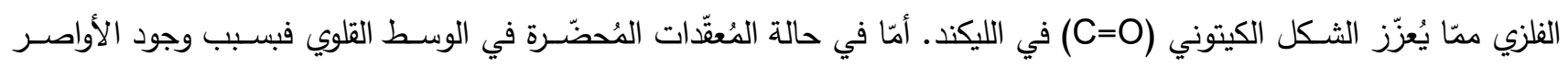

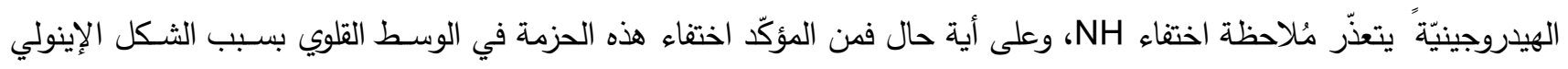

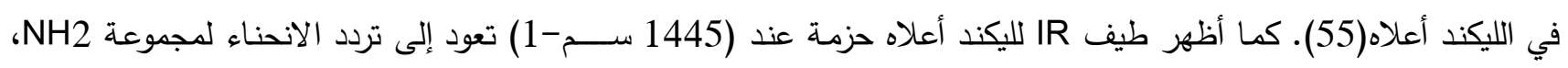

ولوحظ بقاؤها في الموقع نفسه في أطياف جميع المُعقّدات، ممّا يدل على عدم تتاسق هذه المجموعة مع الأيون الفلزي(55).

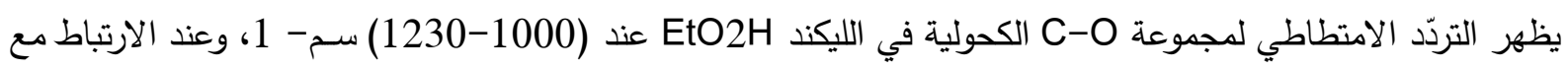
الأيون الفلزي تزاح نحو تردد أعلى (1105- 1172)، هذا يدل على ارتباطها مع الأيون الفلزي. ويظهر التردد الامتطاطي لمجموعة

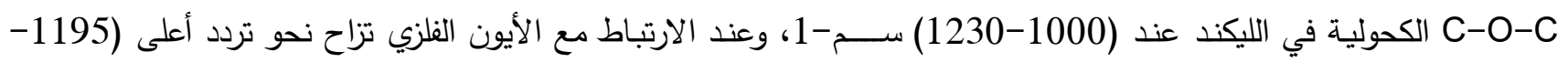

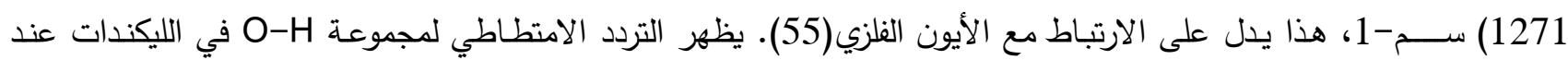

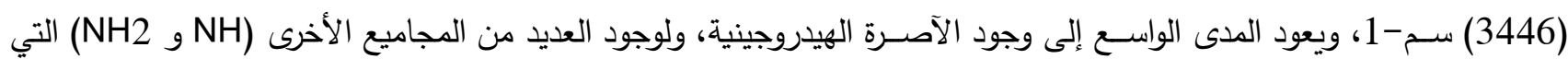

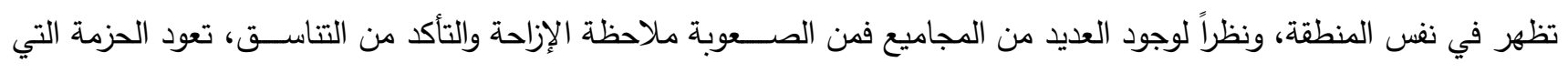
ظهرت في الموقع 1330 ســ-1 إلى تردد الانحناء لمجموعة OH الكحولية، وفي المعقدات لوحظ إزاحة هذه الحزمة إلى تردد أوطأ 
ممّا يدل على اشتراك هذه المجموعة في التناسق مع الأيونات الفلزية(55). عند قياس طيف الأشتعة تحت الحمراء للمُعقدات الفلزيّة

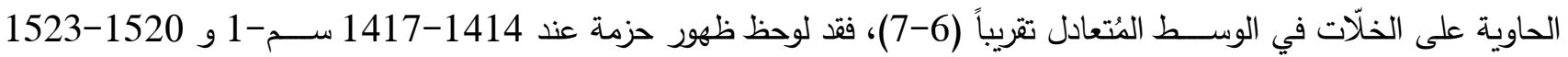

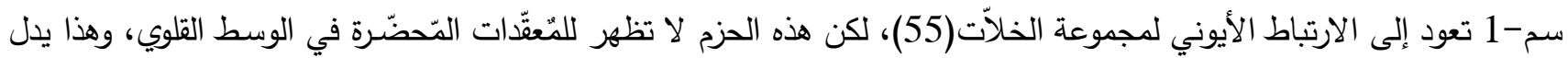

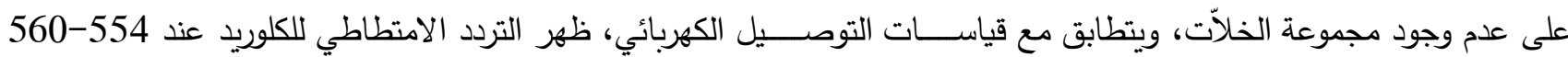

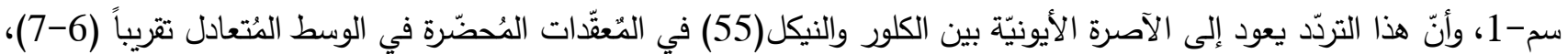

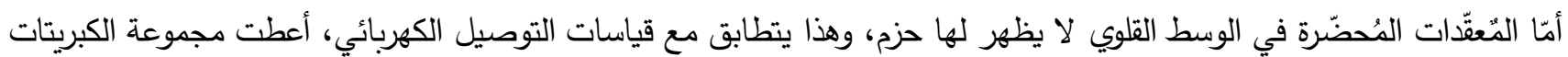

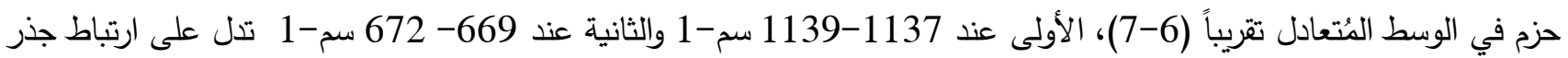

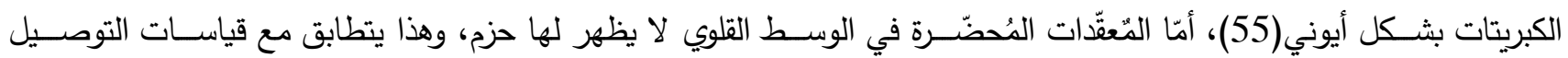
الكهربائي، تعطي مجموعة M-N حزمة عند تردد 409-485 سـ-1 ومجموعة M-O تعطي حزمة 510-595 سـم-1 -1 ظهور

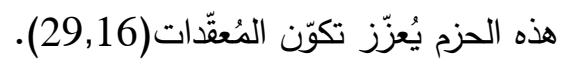

الجدول (5) : نتائج طيف الأشعة تعت الحمراء (سم-10)

\begin{tabular}{|c|c|c|c|c|c|c|c|c|c|c|c|c|c|}
\hline \multirow{2}{*}{$\mathrm{U}_{\mathrm{M}-\mathrm{O}}$} & \multirow[t]{2}{*}{$v_{M-N}$} & \multirow[t]{2}{*}{$\mathrm{v}_{\mathrm{Cl}^{-}}$} & \multirow[t]{2}{*}{$\mathrm{USO}_{\mathrm{SO}}{ }^{2-}$} & \multirow[t]{2}{*}{$\mathrm{v}_{\mathrm{AcO}}{ }^{-}$} & \multirow{2}{*}{$\begin{array}{l}\text { Uo-H; } \\
\text { Un }_{\mathrm{NH} 2} \\
\boldsymbol{\delta}_{\mathrm{NH} 2}\end{array}$} & \multicolumn{2}{|c|}{ Alcohol } & \multicolumn{2}{|c|}{ Oxim } & \multicolumn{3}{|c|}{ Semi } & \multirow[b]{2}{*}{$ت$} \\
\hline & & & & & & $U_{C-O-C}$ & $U_{\mathrm{C}-0 .}$ & $\begin{array}{l}v_{O-H} \\
v_{N O}\end{array}$ & $\mathrm{U}_{\mathrm{C}=\mathrm{N}}$ & $\mathrm{v}_{\mathrm{N}-\mathrm{H}}$ & $\begin{array}{c}U_{\mathrm{C}=0} \\
\text { or } U_{\mathrm{C}-\mathrm{O}}\end{array}$ & $\mathrm{U}_{\mathrm{C}=\mathrm{N}}$ & \\
\hline- & - & - & - & - & 1445 & - & - & - & - & 3211 & 1730 & 1678 & ASCH \\
\hline- & - & - & - & - & - & - & - & $\begin{array}{c}3109-3184 \\
989\end{array}$ & 1662 & - & - & - & $\mathrm{DO}_{\mathrm{X}} \mathrm{PH}_{2}$ \\
\hline- & - & - & - & - & 3446 & $1000-1230$ & $\begin{array}{l}1000- \\
1230\end{array}$ & - & - & - & - & - & $\mathrm{EtO}_{2} \mathrm{H}$ \\
\hline $\begin{array}{c}515,535 \\
590 \\
\end{array}$ & $\begin{array}{c}410,420 \\
460 \\
\end{array}$ & - & - & $\begin{array}{l}1417 \\
1523 \\
\end{array}$ & $\begin{array}{c}3391 \\
1445 \\
\end{array}$ & 1270 & 1145 & $\begin{array}{c}3045-3205 \\
994 \\
\end{array}$ & 1594 & 3205 & 1693 & 1628 & 1 \\
\hline $\begin{array}{c}\mathbf{5 1 7 , 5 3 7} \\
\mathbf{5 8 5}\end{array}$ & $\begin{array}{c}413,425 \\
463\end{array}$ & - & - & $\begin{array}{l}1414 \\
1520\end{array}$ & $\begin{array}{l}3395 \times 1 \\
445\end{array}$ & 1271 & 1143 & $\begin{array}{c}\text { 3041-3206 } \\
999\end{array}$ & 1592 & 3206 & 1682 & 1623 & 2 \\
\hline $\begin{array}{c}\mathbf{5 1 6 , 5 3 3} \\
\mathbf{5 8 7}\end{array}$ & $\begin{array}{c}411,422 \\
462\end{array}$ & - & - & $\begin{array}{l}1416 \\
1522\end{array}$ & $\begin{array}{c}3390 \\
1445\end{array}$ & 1268 & 1140 & $\begin{array}{c}\text { 3046-3204 } \\
999\end{array}$ & 1600 & 3204 & 1690 & 1625 & 3 \\
\hline $\begin{array}{c}530,556 \\
595\end{array}$ & $\begin{array}{c}420,450 \\
478\end{array}$ & - & - & - & $\begin{array}{c}3391 \\
1445 \\
\end{array}$ & 1262 & 1137 & $\begin{array}{c}\text { 3059-3192 } \\
995\end{array}$ & 1605 & - & $\begin{array}{r}- \\
1118\end{array}$ & 1621 & 4 \\
\hline $\begin{array}{c}\mathbf{5 3 2 , 5 5 4} \\
\mathbf{5 9 0}\end{array}$ & $\begin{array}{c}421,449 \\
475\end{array}$ & - & - & - & $\begin{array}{c}3396 \\
1445\end{array}$ & 1263 & 1139 & $\begin{array}{c}3051-3193 \\
999\end{array}$ & 1594 & - & $\begin{array}{c}- \\
1114\end{array}$ & 1623 & 5 \\
\hline $\begin{array}{c}510,542 \\
580\end{array}$ & $\begin{array}{c}419,433 \\
472\end{array}$ & 560 & - & - & $\begin{array}{l}3400 \\
1445\end{array}$ & 1264 & 1105 & $\begin{array}{c}\text { 3061-3097 } \\
999\end{array}$ & 1588 & 3211 & 1685 & 1626 & 6 \\
\hline $\begin{array}{c}512,540 \\
575 \\
\end{array}$ & $\begin{array}{c}422,430 \\
470 \\
\end{array}$ & 554 & - & - & $\begin{array}{l}3399 \\
1445 \\
\end{array}$ & 1263 & 1107 & $\begin{array}{c}3064-3098 \\
997 \\
\end{array}$ & 1589 & 3210 & $\begin{array}{c}1687 \\
-\end{array}$ & 1625 & 7 \\
\hline $\begin{array}{c}\mathbf{5 1 5 , 5 3 5} \\
\mathbf{5 8 2} \\
\end{array}$ & $\begin{array}{c}420,431 \\
468\end{array}$ & 558 & - & - & $\begin{array}{l}3398 \\
1445 \\
\end{array}$ & 1266 & 1106 & $\begin{array}{c}\text { 3066-3097 } \\
995\end{array}$ & 1590 & 3207 & $\begin{array}{c}1685 \\
-\end{array}$ & 1627 & 8 \\
\hline $\begin{array}{c}520,542 \\
588 \\
\end{array}$ & $\begin{array}{c}425,433 \\
485 \\
\end{array}$ & - & - & - & $\begin{array}{l}\mathbf{3 4 1 2} \\
1445 \\
\end{array}$ & 1257 & 1172 & $\begin{array}{c}\text { 3059-3202 } \\
995 \\
\end{array}$ & 1603 & - & $\begin{array}{c}- \\
1112 *\end{array}$ & 1625 & 9 \\
\hline $\begin{array}{c}523,540 \\
586\end{array}$ & $\begin{array}{c}423,435 \\
482\end{array}$ & - & - & - & $\begin{array}{l}3410 \\
1445\end{array}$ & 1259 & 1169 & $\begin{array}{c}\text { 3035-3203 } \\
998\end{array}$ & 1605 & - & ${ }^{-}$ & 1624 & 10 \\
\hline $\begin{array}{c}\mathbf{5 3 0 , 5 5 7} \\
\mathbf{5 8 5} \\
\end{array}$ & $\begin{array}{c}411,420 \\
459\end{array}$ & - & $\begin{array}{c}670 \\
1139 \\
\end{array}$ & - & $\begin{array}{l}3392 \\
1445 \\
\end{array}$ & 1263 & 1147 & $\begin{array}{c}3064-3208 \\
899 \\
\end{array}$ & 1605 & 3208 & $\begin{array}{c}1693 \\
- \\
\end{array}$ & 1624 & 11 \\
\hline $\begin{array}{c}528,558 \\
586\end{array}$ & $\begin{array}{c}409,435 \\
470\end{array}$ & - & $\begin{array}{c}669 \\
1137 \\
\end{array}$ & - & $\begin{array}{l}3393 \\
1445 \\
\end{array}$ & 1260 & 1142 & $\begin{array}{c}\text { 3065-3210 } \\
999\end{array}$ & 1606 & 3210 & $\begin{array}{c}1695 \\
- \\
\end{array}$ & 1625 & 12 \\
\hline $\begin{array}{c}525,556 \\
589\end{array}$ & $\begin{array}{c}413,432 \\
469\end{array}$ & - & $\begin{array}{c}672 \\
1138\end{array}$ & - & $\begin{array}{l}3395 \\
1445\end{array}$ & 1263 & 1144 & $\begin{array}{c}\text { 3035-3208 } \\
998\end{array}$ & 1604 & 3208 & $\begin{array}{c}1693 \\
-\end{array}$ & 1622 & 13 \\
\hline $\begin{array}{c}\mathbf{5 1 5 , 5 3 0} \\
572\end{array}$ & $\begin{array}{c}410,432 \\
462\end{array}$ & - & - & - & $\begin{array}{l}3400 \\
1445\end{array}$ & 1198 & 1130 & $\begin{array}{c}\text { 3057-3211 } \\
995\end{array}$ & 1603 & - & $\begin{array}{c}- \\
111 * 1\end{array}$ & 1621 & 14 \\
\hline $\begin{array}{c}519,532 \\
569\end{array}$ & $\begin{array}{c}413,430 \\
460\end{array}$ & - & - & - & $\begin{array}{l}\mathbf{3 4 0 4} \\
1445\end{array}$ & 1195 & 1133 & $\begin{array}{c}\text { 3055-3209 } \\
995\end{array}$ & 1605 & - & $1113^{*}$ & 1623 & 15 \\
\hline
\end{tabular}



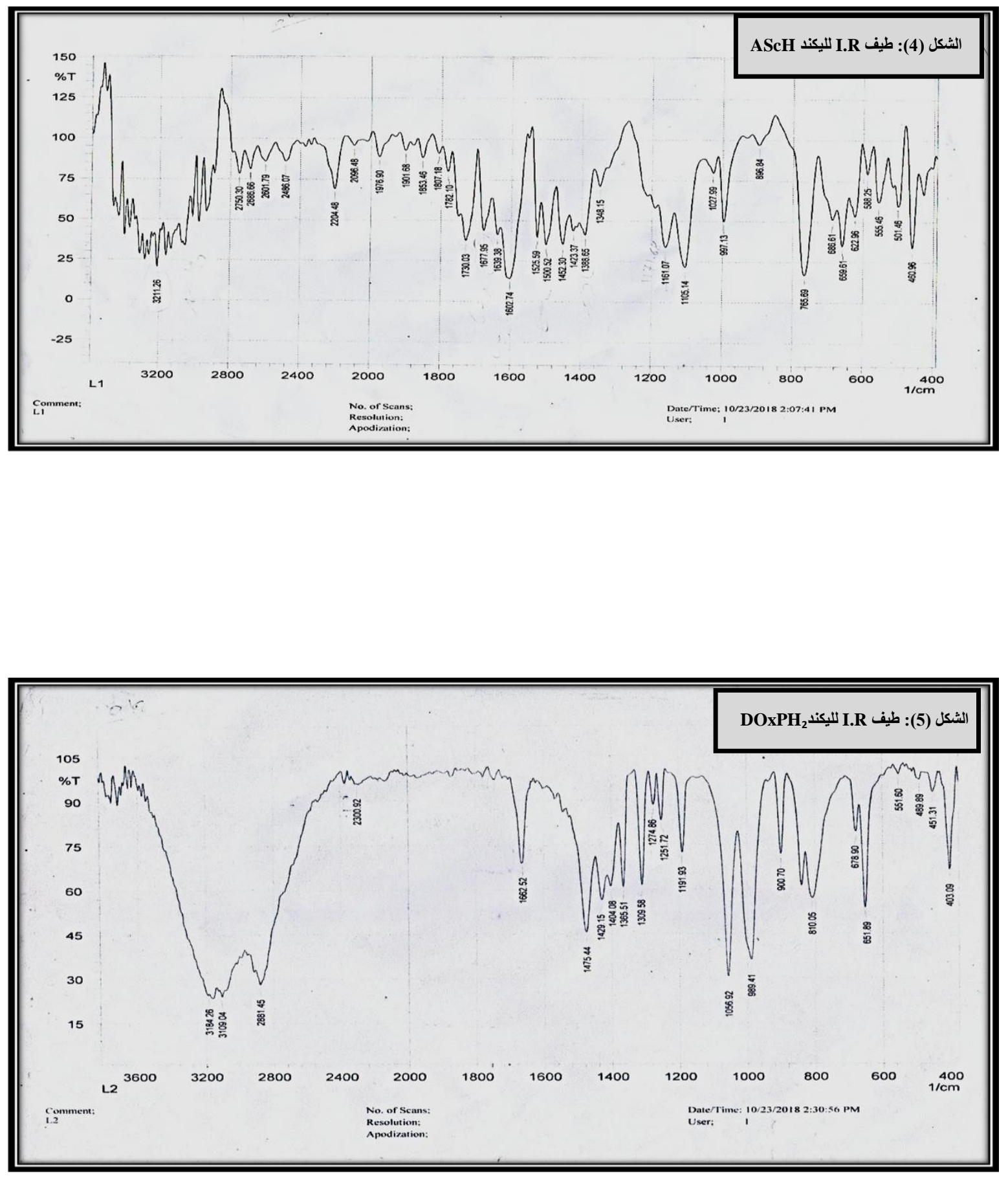

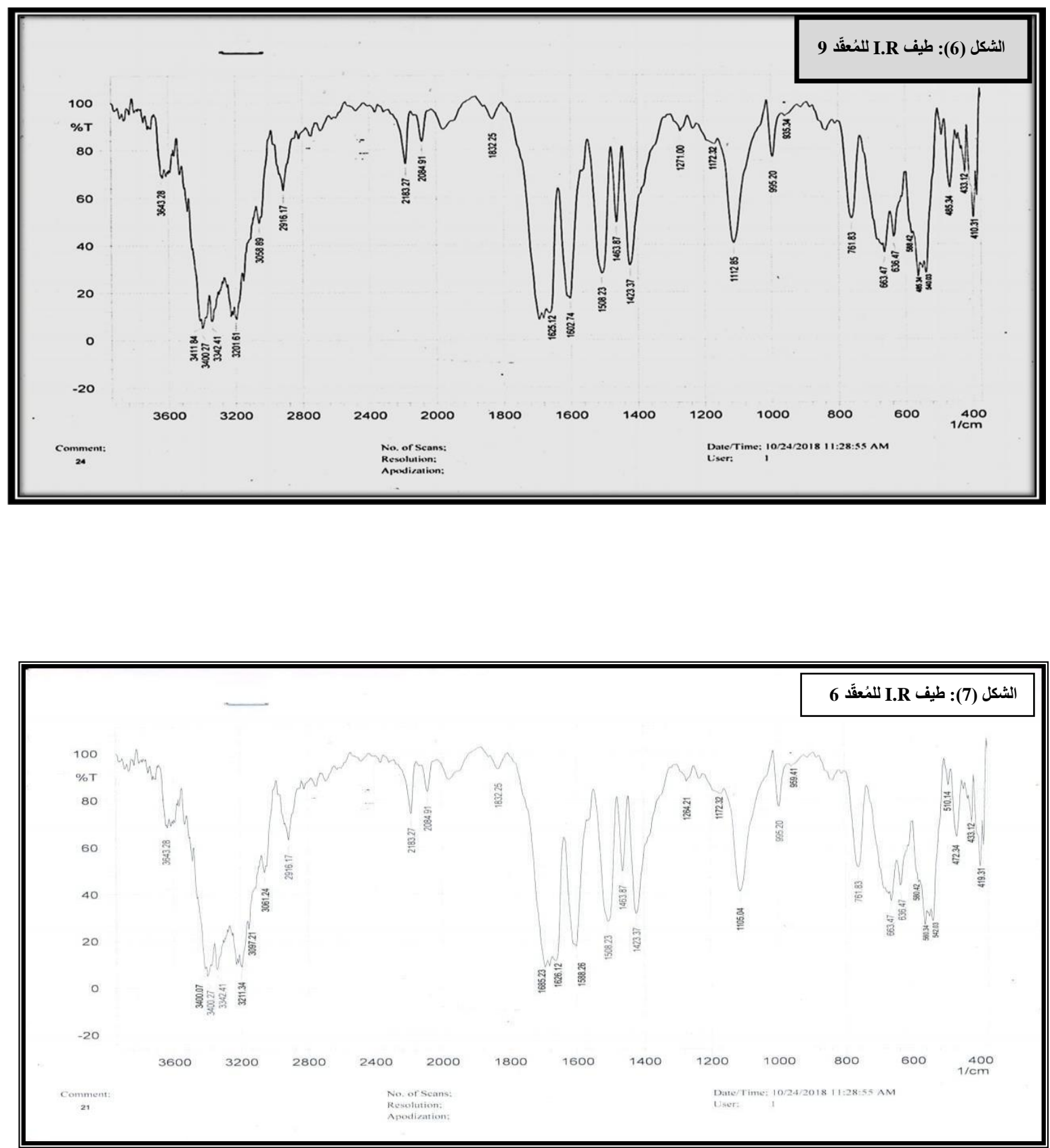

إنّ جهاز طيف الكتلة يعمل على تأيين الجُزيئات وكذلك أجزائها في الحالة الغازيّة، ويُمكن بواسـطته إيجاد النســب للمواد

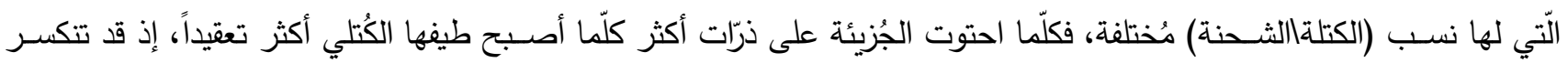

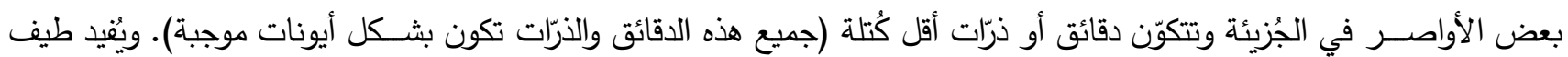

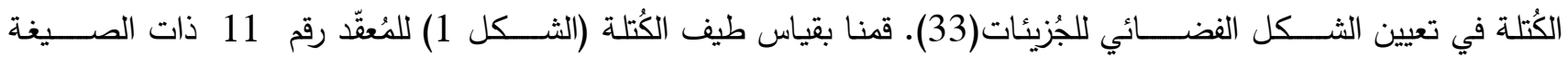
[المُبينّة في الثكل (1) ولقد جاءت النتائج مُطابقة لتوقِعاتنا. 


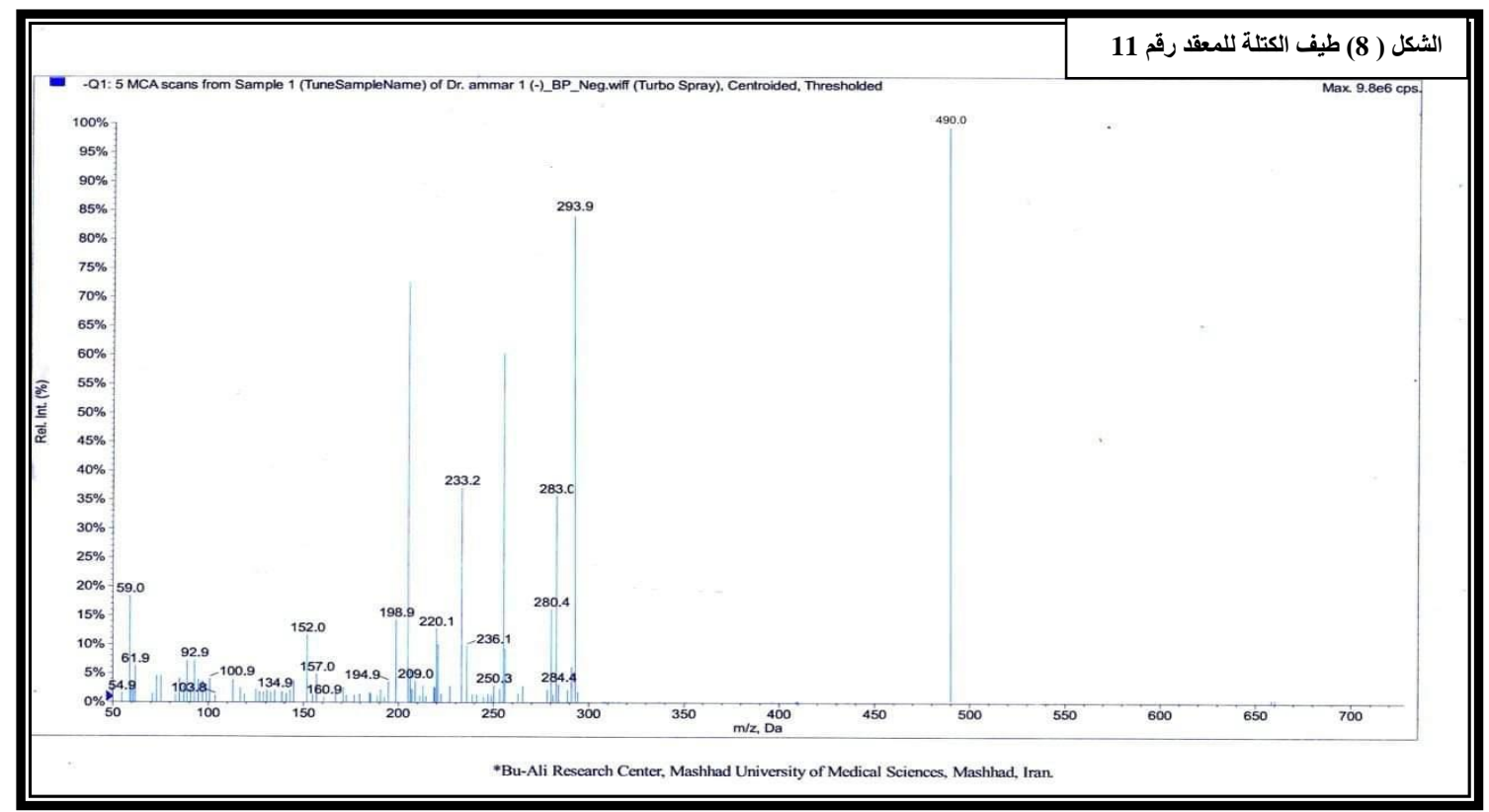

شُعِعت جميع المركبات قيد الدرس بأثعِّة الليزر لمدّة زمنيّة انحصرت من نصف ساعة إلى ساعتين ونصف، لاحظنا أنّ

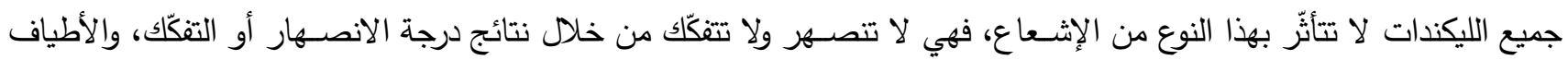

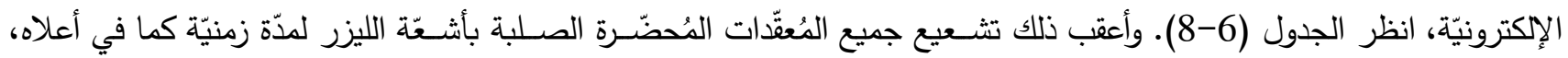

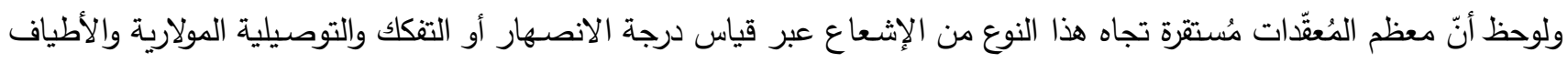

الجدول (6) : نتائج تثعيع الليكندات بأشقّة الليزر

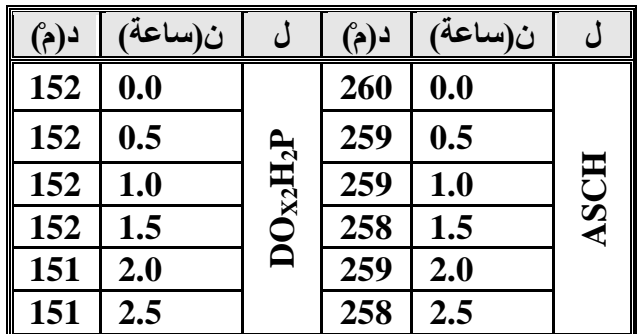




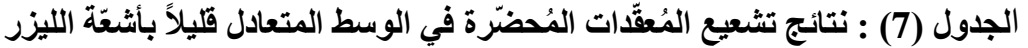

\begin{tabular}{|c|c|c|c|c|c|c|c|c|c|c|c|c|c|c|}
\hline $\begin{array}{c}U_{1} \\
\left({ }^{1-1}\right)\end{array}$ & ص & (2) & (ساعة) & المعقد & $\begin{array}{c}v_{1} \\
\left({ }^{1-1}\right)\end{array}$ & ص & (p) & (ساعة) & المققد & $\begin{array}{c}U_{1} \\
\left({ }^{1-2}\right) \\
\end{array}$ & ص & $\dot{a}$ & (ساعة) & المعقد \\
\hline 10193.68 & 154 & $195 *$ & $\begin{array}{c}0.0 \\
\end{array}$ & \multirow{6}{*}{3} & 10193.68 & 156 & $195 *$ & 0.0 & \multirow{6}{*}{2} & 10193.68 & 155 & $195 *$ & 0.0 & \multirow{6}{*}{1} \\
\hline 10193.68 & 156 & 194* & 0.5 & & 10193.68 & 154 & $195 *$ & 0.5 & & 10193.68 & 156 & $195 *$ & 0.5 & \\
\hline 10193.68 & 156 & 195* & 1.0 & & 10193.68 & 154 & $196 *$ & 1.0 & & \begin{tabular}{|l|l|l|}
10193.68 \\
\end{tabular} & 154 & 196* & 1.0 & \\
\hline 10193.68 & 155 & $195 *$ & 1.5 & & 10193.68 & 156 & $196 *$ & 1.5 & & 10193.68 & 156 & 194* & 1.5 & \\
\hline 10193.68 & 155 & $196 *$ & 2.0 & & 10193.68 & 155 & $195 *$ & 2.0 & & 10193.68 & 156 & $196^{*}$ & 2.0 & \\
\hline 10193.68 & 155 & 196* & 2.5 & & 10193.68 & 155 & 194* & 2.5 & & 10193.68 & 154 & 195* & 2.5 & \\
\hline 10193.68 & 168 & 200* & 0.0 & \multirow{6}{*}{8} & $\begin{array}{r}10193.68 \\
\end{array}$ & 170 & 200* & 0.0 & \multirow{6}{*}{7} & 10193.68 & 169 & 200* & 0.0 & \multirow{6}{*}{6} \\
\hline 10193.68 & 169 & 200* & 0.5 & & 10193.68 & 170 & 201* & 0.5 & & 10193.68 & 169 & 200* & 0.5 & \\
\hline 10193.68 & 170 & 199* & 1.0 & & \begin{tabular}{|l|l|l|}
10193.68 \\
\end{tabular} & 168 & 201* & 1.0 & & 10193.68 & 168 & 200* & 1.0 & \\
\hline 10193.68 & 170 & 199* & 1.5 & & \begin{tabular}{|l|l|l|}
10193.68 \\
\end{tabular} & 170 & 200* & 1.5 & & 10193.68 & 169 & 199* & 1.5 & \\
\hline 10193.68 & 169 & 200* & 2.0 & & 10193.68 & 169 & 199* & 2.0 & & 10193.68 & 168 & 199* & 2.0 & \\
\hline 10193.68 & 169 & 201* & 2.5 & & 10193.68 & 168 & 201* & 2.5 & & 10193.68 & 170 & 201* & 2.5 & \\
\hline 10101.01 & 165 & 243 & 0.0 & \multirow{6}{*}{13} & 10101.01 & 162 & 243 & 0.0 & \multirow{6}{*}{12} & 10101.01 & 163 & 243 & 0.0 & \multirow{6}{*}{11} \\
\hline 10101.01 & 165 & 244 & 0.5 & & 10101.01 & 163 & 243 & 0.5 & & 10101.01 & 163 & 244 & 0.5 & \\
\hline 10101.01 & 165 & 243 & 1.0 & & 10101.01 & 162 & 244 & 1.0 & & 10101.01 & 162 & 243 & 1.0 & \\
\hline 10101.01 & 163 & 243 & 1.5 & & 10101.01 & 163 & 244 & 1.5 & & 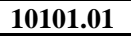 & 165 & 241 & 1.5 & \\
\hline 10101.01 & 162 & 243 & 2.0 & & 10101.01 & 165 & 243 & 2.0 & & 10101.01 & 162 & 241 & 2.0 & \\
\hline 10101.01 & 162 & 241 & 2.5 & & 10101.01 & 165 & 243 & 2.5 & & 10101.01 & 165 & 244 & 2.5 & \\
\hline
\end{tabular}

الجدول (8) : نتائج تثعيع المُعقّات المُحضرّة في الوسط القلوي بأثقعة الليزر

\begin{tabular}{|c|c|c|c|c|c|c|c|c|c|}
\hline U1 & ص & (a) 1 & ن (ساعة) & رقم المعقد & (سم-1) & ص & (p) 1 & ن (ساعة) & رقم المعقد \\
\hline 10172.94 & 3 & 265 & (2.0 & \multirow{6}{*}{5} & 10172.94 & 2 & 265 & 0.0 & \multirow{6}{*}{4} \\
\hline 10172.94 & 3 & 265 & 0.5 & & 10172.94 & 3 & 265 & 0.5 & \\
\hline 10172.94 & 3 & 265 & 1.0 & & 10172.94 & 3 & 265 & 1.0 & \\
\hline 10172.94 & 3 & 267 & 1.5 & & 10172.94 & 3 & 265 & 1.5 & \\
\hline 10172.94 & 3 & 267 & 2.0 & & 10172.94 & 3 & 267 & 2.0 & \\
\hline 10172.94 & 3 & 267 & 2.5 & & 10172.94 & 3 & 267 & 2.5 & \\
\hline 10172.94 & 8 & 265 & 0.0 & \multirow{6}{*}{10} & 10172.94 & 7 & 265 & 0.0 & \multirow{6}{*}{9} \\
\hline 10172.94 & 8 & 264 & 0.5 & & 10172.94 & 8 & 265 & 0.5 & \\
\hline 10172.94 & 7 & 264 & 1.0 & & 10172.94 & 8 & 265 & 1.0 & \\
\hline 10172.94 & 7 & 264 & 1.5 & & 10172.94 & 8 & 264 & 1.5 & \\
\hline 10172.94 & 7 & 264 & 2.0 & & 10172.94 & 8 & 264 & 2.0 & \\
\hline 10172.94 & 7 & 264 & 2.5 & & 10172.94 & 8 & 264 & 2.5 & \\
\hline 10172.94 & 3 & 265 & 0.0 & \multirow{6}{*}{15} & 10172.94 & 2 & 265 & 0.0 & \multirow{6}{*}{14} \\
\hline 10172.94 & 3 & 265 & 0.5 & & 10172.94 & 2 & 265 & 0.5 & \\
\hline 10172.94 & 3 & 265 & 1.0 & & 10172.94 & 3 & 265 & 1.0 & \\
\hline 10172.94 & 3 & 265 & 1.5 & & 10172.94 & 3 & 265 & 1.5 & \\
\hline 10172.94 & 2 & 265 & 2.0 & & 10172.94 & 3 & 265 & 2.0 & \\
\hline 10172.94 & 2 & 265 & 2.5 & & 10172.94 & 3 & 265 & 2.5 & \\
\hline
\end{tabular}

(Staphylococcus aureus) أظهرت نتائج تقييم الفعَاليّة الحيويّة لليكندات والمُعقّات قيد الدرس على البكتريا المرضيّة و وما مبين في الجدول ادناه (E. coli ) ولاه (Streptococcus specie) 
Journal of Education and Science (ISSN 1812-125X), Vol: 29, No: 1, 2020 (22-42)

الجدول (9): الفعّاليّة مضادّة البكتريا لليكندات و للمُعقّدات

\begin{tabular}{|c|c|c|c|c|c|c|c|c|c|}
\hline \multirow[b]{2}{*}{ المُعقد } & \multicolumn{3}{|c|}{ Streptococcus species } & \multicolumn{3}{|c|}{ Staphylococcus aureus } & \multicolumn{3}{|c|}{ E. coli } \\
\hline & $\begin{array}{r}500 \\
\mu \mathrm{g} / \mathrm{ml}\end{array}$ & $\begin{array}{r}250 \\
\mu \mathrm{g} / \mathrm{ml}\end{array}$ & $\begin{array}{r}125 \\
\mu \mathrm{g} / \mathrm{ml}\end{array}$ & $\begin{array}{r}500 \\
\mu \mathrm{g} / \mathrm{ml}\end{array}$ & $\begin{array}{r}250 \\
\mu \mathrm{g} / \mathrm{ml} \\
\end{array}$ & $\begin{array}{r}125 \\
\mu \mathrm{g} / \mathrm{ml}\end{array}$ & $\begin{array}{c}500 \\
\mu \mathrm{g} / \mathrm{ml}\end{array}$ & $\begin{array}{c}250 \\
\mu \mathrm{g} / \mathrm{ml}\end{array}$ & $\begin{array}{c}125 \\
\mu \mathrm{g} / \mathrm{ml}\end{array}$ \\
\hline ScH A & $\mathbf{R}$ & $\mathbf{R}$ & $\mathbf{R}$ & $\mathbf{R}$ & $\mathbf{R}$ & $\mathbf{R}$ & $\mathbf{R}$ & $\mathbf{R}$ & $\mathbf{R}$ \\
\hline $\mathrm{ACO}_{\mathrm{xH}}$ & $\mathbf{R}$ & $\mathbf{R}$ & $\mathbf{R}$ & $\mathbf{R}$ & $\mathbf{R}$ & $\mathbf{R}$ & $\mathbf{R}$ & $\mathbf{R}$ & $\mathbf{R}$ \\
\hline 1 & $\mathbf{R}$ & $\mathbf{R}$ & $\mathbf{R}$ & $\mathbf{R}$ & $\mathbf{R}$ & $\mathbf{R}$ & $\mathbf{R}$ & $\mathbf{R}$ & $\mathbf{R}$ \\
\hline 2 & $\mathbf{R}$ & $\mathbf{R}$ & $\mathbf{R}$ & $\mathbf{R}$ & $\mathbf{R}$ & $\mathbf{R}$ & $\mathbf{R}$ & $\mathbf{R}$ & $\mathbf{R}$ \\
\hline 3 & $\mathbf{R}$ & $\mathbf{R}$ & $\mathbf{R}$ & $\mathbf{R}$ & $\mathbf{R}$ & $\mathbf{R}$ & $\mathbf{R}$ & $\mathbf{R}$ & $\mathbf{R}$ \\
\hline 4 & $\mathbf{R}$ & $\mathbf{R}$ & $\mathbf{R}$ & $\mathbf{R}$ & $\mathbf{R}$ & $\mathbf{R}$ & $\mathbf{R}$ & $\mathbf{R}$ & $\mathbf{R}$ \\
\hline 5 & $\mathbf{R}$ & $\mathbf{R}$ & $\mathbf{R}$ & $\mathbf{R}$ & $\mathbf{R}$ & $\mathbf{R}$ & $\mathbf{R}$ & $\mathbf{R}$ & $\mathbf{R}$ \\
\hline 6 & $\mathbf{R}$ & $\mathbf{R}$ & $\mathbf{R}$ & $\mathbf{R}$ & $\mathbf{R}$ & $\mathbf{R}$ & $\mathbf{R}$ & $\mathbf{R}$ & $\mathbf{R}$ \\
\hline 7 & $\mathbf{R}$ & $\mathbf{R}$ & $\mathbf{R}$ & $\mathbf{R}$ & $\mathbf{R}$ & $\mathbf{R}$ & $\mathbf{R}$ & $\mathbf{R}$ & $\mathbf{R}$ \\
\hline 8 & $\mathbf{R}$ & $\mathbf{R}$ & $\mathbf{R}$ & $\mathbf{R}$ & $\mathbf{R}$ & $\mathbf{R}$ & $\mathbf{R}$ & $\mathbf{R}$ & $\mathbf{R}$ \\
\hline 9 & $\mathbf{R}$ & $\mathbf{R}$ & $\mathbf{R}$ & $\mathbf{R}$ & $\mathbf{R}$ & $\mathbf{R}$ & $\mathbf{R}$ & $\mathbf{R}$ & $\mathbf{R}$ \\
\hline 10 & $\mathbf{R}$ & $\mathbf{R}$ & $\mathbf{R}$ & $\mathbf{R}$ & $\mathbf{R}$ & $\mathbf{R}$ & $\mathbf{R}$ & $\mathbf{R}$ & $\mathbf{R}$ \\
\hline 11 & $\mathbf{R}$ & $\mathbf{R}$ & $\mathbf{R}$ & $\mathbf{R}$ & $\mathbf{R}$ & $\mathbf{R}$ & $\mathbf{R}$ & $\mathbf{R}$ & $\mathbf{R}$ \\
\hline 12 & $\mathbf{R}$ & $\mathbf{R}$ & $\mathbf{R}$ & $\mathbf{R}$ & $\mathbf{R}$ & $\mathbf{R}$ & $\mathbf{R}$ & $\mathbf{R}$ & $\mathbf{R}$ \\
\hline 13 & $\mathbf{R}$ & $\mathbf{R}$ & $\mathbf{R}$ & $\mathbf{R}$ & $\mathbf{R}$ & $\mathbf{R}$ & $\mathbf{R}$ & $\mathbf{R}$ & $\mathbf{R}$ \\
\hline 14 & $\mathbf{R}$ & $\mathbf{R}$ & $\mathbf{R}$ & $\mathbf{R}$ & $\mathbf{R}$ & $\mathbf{R}$ & $\mathbf{R}$ & $\mathbf{R}$ & $\mathbf{R}$ \\
\hline 15 & $\mathbf{R}$ & $\mathbf{R}$ & $\mathbf{R}$ & $\mathbf{R}$ & $\mathbf{R}$ & $\mathbf{R}$ & $\mathbf{R}$ & $\mathbf{R}$ & $\mathbf{R}$ \\
\hline $\begin{array}{l}\text { Standard } \\
\text { antibiotic }\end{array}$ & \multicolumn{3}{|c|}{$13 \mathrm{~mm}$} & \multicolumn{3}{|c|}{$14 \mathrm{~mm}$} & \multicolumn{3}{|c|}{$12 \mathrm{~mm}$} \\
\hline
\end{tabular}

\section{الاستنتاج}

اعتماداً على نتائج القياسات الكميّة والقياسات الطيفيّة والخصائص الفيزيائيّة وتأثير أشعة الليزر وتتييم الفعّاليّة ضد بكتريا

Staphylococcus aureus و Streptococcus species

1.يتتاسق الليكند AScH مع ايون النيكل(II) في جميع المُعْدات في الوسط المتعادل تقريباً (الدالة الحامضيّة 6-7) من خلال ذرّة

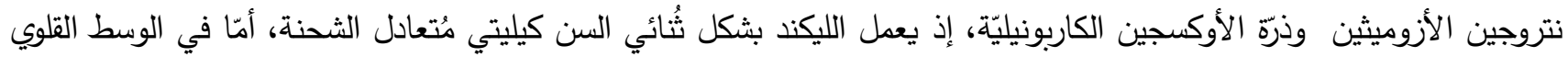

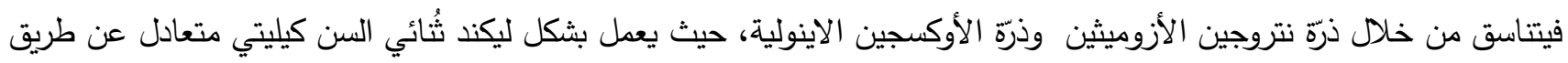
تحوله من هيئة الكيتو إلى هيئة ألاينول كما هو مُبيّن أدناه:

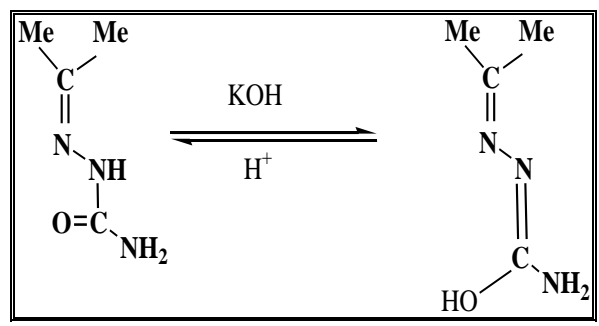

• يتتاسق ليكند DOXPH2 في جميع المُعِقدات في الوسط المتعادل تقريباً (الدالة الحامضيّة 6-7) من خلال ذرّتي نتروجين

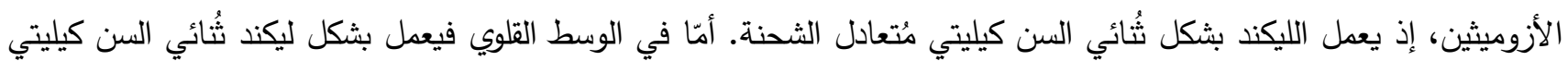

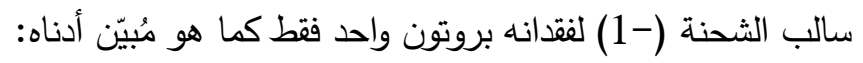




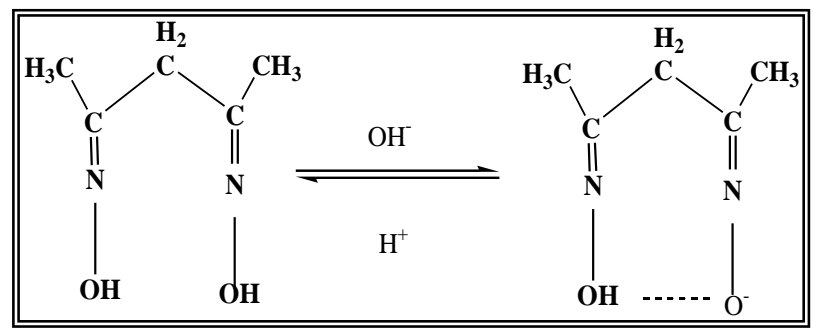

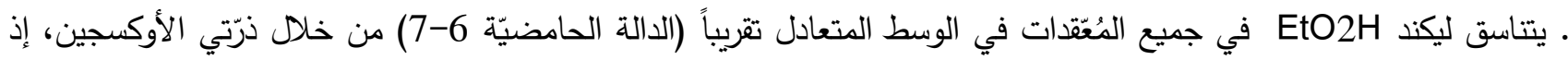
يعمل الليكند بثكل ثُنائي السن كيليتي مُتعادل الثحنة. أمّا في الوسط القلوي فيعمل بشكل ليكند ثُنائي السن كيليتي سالب الثحنة

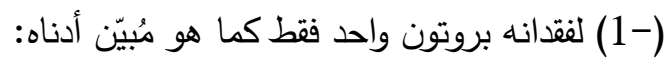

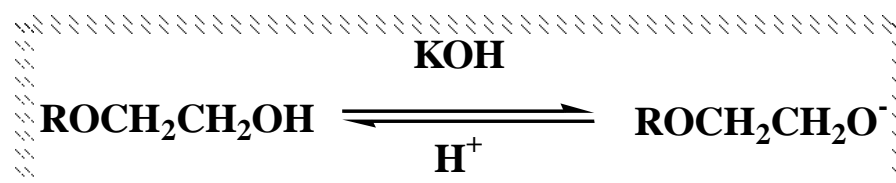

\section{$\mathrm{R}=\mathrm{CH}_{\mathbf{3}} \mathrm{CH}_{\mathbf{2}}$}

4. لا تتناسق الأيونات اللاعضوية (CH3COO و و و $\mathrm{Cl}$ و بثكل أيوني ل 5. المُعِقدات الناتجة في الوسط المتعادل تقريباً تكون بثكل أيونية موجبة (2+2) صيغتها 6. المُعْقدات الناتجة في الوسط القلوي تكون مُتعادلة صيغتها ل

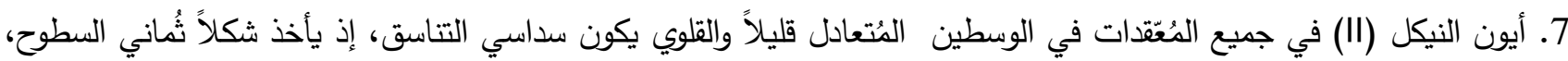
كما هو مُبيّن بالأشكال أدناه

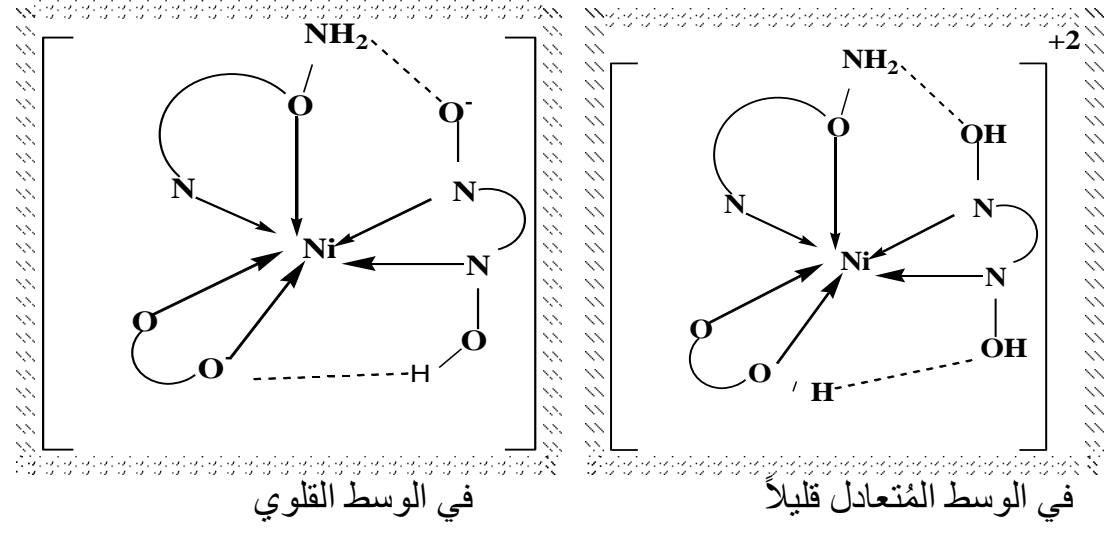



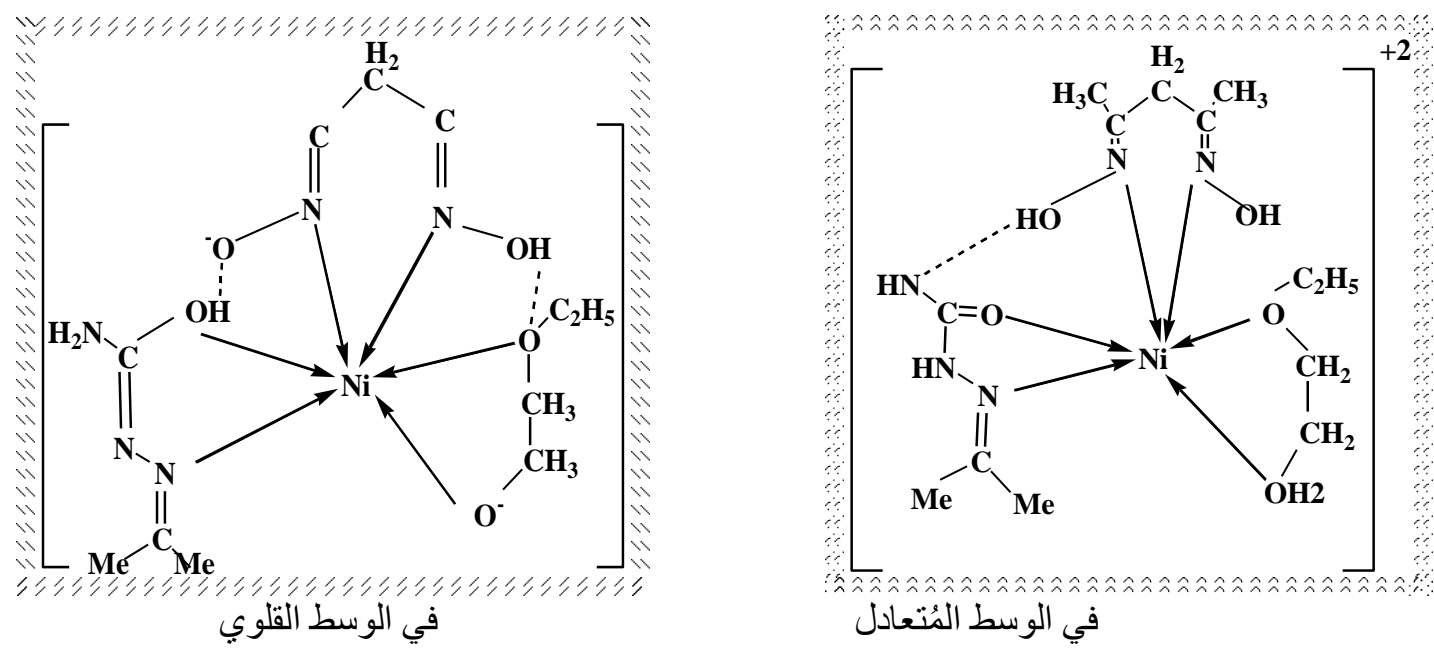

$$
\text { الثكل (9) يبيّن اشكال المُعقّات في الوسطين المتعادل والقلوي }
$$

8. اعتماداً إلى نتائج درجة الإنصهار او التفكك والتوصيلية الكهربائية وأطياف الأشعة فوق البنفجية - المرئية والتشعيع بالليزر

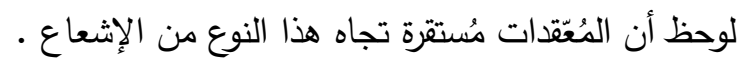

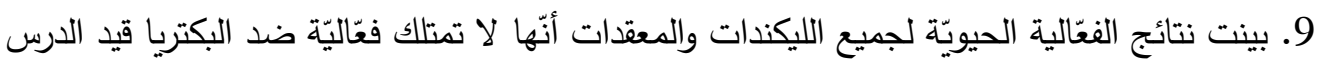

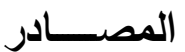

1. A. Al-Tharra; Nickel, J.A.E.C.S., 105, 45-47, This Knowledge is published in ; J. ANL -October (2001).

2. F.A. Cotton and G. Wilkinson; Advanced Inorganic Chemistry; Inter science, New York, $5^{\text {th }}$ ed., $597,599,680,689,702,766(1988)$.

3. G. Wilkinson, R.D. Gillard and J.A. McClevrty; Comprehensive Coordination Chemistry; Pergamon Press, Oxford, England, 5, 46-49 (1987).

4. http://ar.wikipedia.org. (2018)

5. A. Stwertka; "A Guide To The Elements", Oxford Univ. Press Inc., New York, Rev. ed., 96-98 (1998) .

6. M.Hijazi, "Principles of Dermatology", Chapter 25 Eczema resulting from manufactured materials.

7. Toyo Rayon Com.; "Lactams", Jappan Appl., 14 (1966); Chem. Abst., 76, 59481e (1972).

8. Chemical Land 21.com.; "General Description and Applica-tion of Benzaldoxime", (2005). 9.Aaron Rowe, New Nerve Gas Antidotes. (http://blog.wired.com/Wiredsience/2007/11/building-abett.htm); Wired(Magazine) [[2007-11-27 ]].

10. M. Windholz; "The Merck Index", Merck and Co. Inc., Rahway, N. J., USA, $11^{\text {th }}$ ed.(1989). 
11. J. Kassa; J. Tox.- Clinical Toxicology, 40, 803 (2002) http://dx.doi.org/10.1081/CLT-120015840).

12. H. Kalasz, M.Y. Hasan, R. Sheen, k. Kuca, G. Petroianu, K. Ludanyi, A. Gergely and K. Tekes, J. Bio. Anal. Chem., 385, 1062-1067 (2007).

13. H. Bati, A. Zülfikaroğlu , M. Tas, O. Andac and W.T.A. Harrison; Acta Cryst., E61, m2033-m2035 (2005).

14. A. Shokrollahi, M. Ghaedi, H.R. Rajabi, M.S. Niband; Spectrochim. Acta A Mol. Biomol. Spectrosc., 15;71(2), 655-62 (2008).

15. M. Kurtoglu; J. Serb. Chem. Soc.; 75(9), 1231-1239 (2010).

16. L. Martínez, J.S. Gancheff, F.E. Hahn, R.A. Burrow, R. González, C. Kremer, R. Chiozzone; Spectrochim. Acta A Mol Biomol Spectrosc.; 15(105) 439-445 ( 2013).

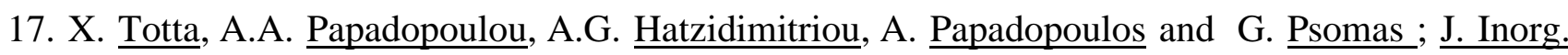
Biochem.; 145, 79-93 (2015).

18. D. Basu, T. J. Woods and Th. B. Rauchfuss; Dalton Trans., 47, 7256-7262 (2018).

19. E.R. Garbelini, M. Hörner, V.F. Giglio, A.H. da Silva, A. Barison and F.S. Nunes; Z. Anorg. Allg. Chem.; 635(8), 1236-1241(2009).

20. J.D. Patel; RASĀYAN J. Chem.; 3(4) 625-630 (2010).

21. M.M. Hania ; RJPBCS; 2(4) 12-17 (2011).

22. K. Sapna, N.K. Sharma and S. Kohli; Intern. J. Sci. \& Eng. Res.; 4(9) 15- 21 (2013).

23. M.H. Shih, Y.Y. Xu, Y.S. Yang and T.T. Lin; Molecules; 20(3) 5184- 5201 (2015).

24. S. Geol, S. Chandra and S. D. Dwivedi, J. Saudi Chem. Soc.; 20 ,651-660 (2016).

25. B.A. Salah, A. Tkandiand, M.G. Abd El-Nassa Res. Rev. J. Chem; 7(1) 38-48 (2018).

26. https://doi.org/10.1080/10915810290096388; International Journal of Toxicology; 21, 11, 2002.

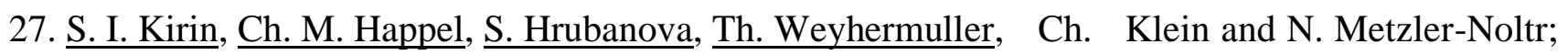
Dalton Frans.; 1201-1207 (2004).

28. O.M.I. Adly; Spectrochim. Acta A Mol. Biomol. Spectrosc., 79(5), 1295-1303 (2011). 
29. P.R. Shirode; Chem Sci Trans.; 1(2), 396-400 (2012)

30. B. Jain, M. Verma and S. Malik; Der Chemica Sinica; 5(1) 77-80 (2014)

31. Z.F. Dawood and N.A.K. Al-neami; مجلة بغداد للعلوم; 10(3) 509-524(2013).

32. Z.F. Dawood and M. A.A. Shaheen; مجلة بغداد للعلوم 11(2) 358-370 (2014)

33. Z.F. Dawood and R. R. A. Al-Bustani; IJST; 12(3) (2017).

34. C.O. Kappe; "Controlled microwave heating in modern organic synthesis"; Angew Chem. Int. Ed., 43, 6250-6284. (2004).

35. G. Rossu and J.A. Pearce; Foundations and industrial applications of microwave and radio frequency fields; John Wiley and Sons, Chichester-New York -Brisbane Toronto - Singapore (1995) .

36. P. Ali, P. Ramakanth and J. Meshram; J. Coord. Chem.; 63(2), 323-329 (2010).

37. G. Chattopadhyay and P.S. Ray; Cheminform; 43, 29(2012).

38. Wikipedia ; Ultrasound(2018).

39. R.K. Shula and A. Sahu; Der Pharma chemical; 5(5)291-295(2013).

40. I. Sheikhshoaie and Z. Tohidiyana; CJBAS; 03(05),164-170 (2015).

41. S. Jadhav, S. KuharuiandS.Qnadri, J. Chem.\&Cheml.Sci,5(6),311-316(2015).

42.I.J.Al-Khatib, A. M. Hamza and E.J.Abdul Jabbar ; Journal of Al-Anbar University for Pure Sciences. (3) 11 61-56 (2017).

43.A. E.A.Saeid. "Free Membership. Foundations of photochemistry and lasers". University Book House. Al Ain, United Arab Emirates. Chapter VII, pp. 143-161 (2003).

44. A.T.Al-Kinani, General Physics, Dar Al-Handasiyah Press, Dar Al-Fajr for Publishing and Distribution, Cairo, 513-492 (2009).

45. M.M. Nasr, M.A. Gondal and Z.H. Yamnai; Fourth Saudi Sci. Conf.; March 21-24 th; 129 (2010). 46. F. Morishita and T. Kojima; Analyt. Lett., 6(3), 179-184 (1973).

47. Z.M.J.M. Al-Deleme; Preparation and Characterization of New Nickel(II) Complexes with Mixed Ligands and Evaluation of Their Antibacterial Activity and effect of Laser on Them, M. Sc. Thesis, Mosul Univ., Educ. Coll., Mosul-Iraq (2013).

48. A.I. Vogel; "Textbook of Practical Organic Chemistry"; Long-man Green, London, $3^{\text {rd }}$ ed., 847, 344 (1964). 
49. A.I. Vogel, "Textbook of Quantitative Inorganic Analysis" Longman Green, London, $3^{\text {rd }}$ ed. (1987).

50. W. J. Geary; Coord. Chem. Rev.; 7, 81 (1981).

51. C. Georg and L. Paul; Copper or nickel complexes of ligands containing an azomethine, semicarbozone or hydrazone moiety; United State Patent 4775747 (1987); http://www.Freepateatsonline.com.

52. J.E. Haheey; Inorganic Chemistry, Harper and Row Publisher, Inc., New York, $2^{\text {nd }}$. Ed.,286 (1978).

53. D. Sutton; Electronic Spectra of Transition metal Complexes; McGrow-Hill Publishing Co., London, 11-21, 146-149 (1968).

54. B.P. Lever; Electronic spectra of some transition metal Complexes; J. Chem. Edu.;45,711-712. (1968)

55. K. Nakamoto; Infrared and Raman Spectra of Inorganic and Coordination Compound; John Wily and Sons, New York, $3^{\text {rd }}$. ed., 166-322 (1976).

56. C. Demetgül; "Synthesis and Characterization of Oxime Compounds immobilized to solid support and their metal complexes", Ph. D. Thesis, Chem. Dept., Institute of Natural and Applied Sci., Cukurova Univ. (2008).

57. I. Demir, M. Bayrakei, K. Mutlu and A.I. Pekacar; Acta Chim. Solv., 55, 120-124 (2008).

58. P.K. Singh, J.K. Koacher and J.P. Tandon; J. Inorg. Nucl. Chem.; 43, 1775 (1981). 